\title{
Frequency Energy Plots of Dynamical Systems Attached to Piecewise Nonlinear Energy Sink
}

\author{
Mohammed Ameen Ameen Al Shudeifat ( $\nabla$ mohd.shudeifat@ku.ac.ae ) \\ Khalifa University of Science Technology https://orcid.org/0000-0002-7973-2559

\section{Adnan Salem Saeed} \\ Khalifa University of Science Technology - Abu Dhabi Campus: Khalifa University of Science and \\ Technology
}

\section{Research Article}

Keywords: Nonlinear energy sink, Piecewise-Linear Nonlinearities, Dead-zone nonlinearities, Frequency energy plot.

Posted Date: March 31st, 2021

DOl: https://doi.org/10.21203/rs.3.rs-252523/v1

License: (9) This work is licensed under a Creative Commons Attribution 4.0 International License. Read Full License 


\title{
Frequency Energy Plots of Dynamical Systems Attached to Piecewise Nonlinear Energy Sink
}

\author{
Mohammad A. AL-Shudeifat \\ Aerospace Engineering, Khalifa University of Science and Technology, \\ Abu Dhabi,127788, UAE, mohd.shudeifat@ku.ac.ae \\ Adnan S. Saeed \\ Aerospace Engineering, Khalifa University of Science and Technology, \\ Abu Dhabi,127788, UAE, adnan.saeed@ku.ac.ae
}

\begin{abstract}
The frequency-energy plots (FEPs) of two-degree-of-freedom linear structures attached to piecewise nonlinear energy sink (PNES) are generated here and thoroughly investigated. This study provides the FEP analysis of such systems for further understanding of nonlinear targeted energy transfer (TET) by the PNES. The attached PNES incorporates a symmetrical clearance zone of zero stiffness content about its equilibrium position where the boundaries of the zone are coupled with linear structure by linear stiffness elements. In addition, linear viscous damping is selected to be continuous during PNES mass oscillation. The underlying nonlinear dynamical behaviour of the considered structure-PNES systems is investigated by generating the fundamental backbone curves of the FEP and the bifurcated subharmonic resonance branches using numerical continuation methods. Accordingly, interesting dynamical behaviour of the nonlinear normal modes (NNMs) of the structure-PNES system on different backbones and subharmonic resonance branches has been observed. In addition, the imposed wavelet transform frequency spectrums on the FEPs have revealed that the TET takes place where it is dominated by the nonlinear action of the PNES.
\end{abstract}

Keywords: Nonlinear energy sink, Piecewise-Linear Nonlinearities, Dead-zone nonlinearities, Frequency energy plot.

\section{Introduction}

Small- and large-scale structural and dynamical systems could be subjected to sudden vibrations caused by wind gusts, blasts, earthquakes, or others, which could impose catastrophic damages to such systems. Hence, various active, semi-active and passive linear and nonlinear dynamical vibration absorbers have been 
extensively investigated for vibration mitigation through dissipating significant portion of seismic or impulsive energy in a rapid manner. Although passive linear vibration absorbers have long been considered as possible solutions for vibration mitigation, they are single-mode and single-frequency tuned attachments where their operation is narrowband. On the other hand, nonlinear vibration absorbers, known as nonlinear energy sinks (NESs), are systems with essentially nonlinear coupling force that significantly alters the global dynamics of the integrated system. Therefore, such systems do not follow preferential resonance frequency where they alternatively possess energy-frequency dependence to tune themselves to a broadband natural frequencies range. This makes them capable to achieve broadband passive energy transfer and dissipation through cascades of resonance captures. Consequently, the NES is considered as a passive frequency-energy adaptable vibration absorber that provides similar properties as active, semi-active and hybrid control systems.

The NESs are usually attached to small and large-scale dynamical structures for rapid vibration suppression which saves structures from unexpected vibrationinduced damages. Therefore, application of NESs has gained rapid and extensive research interest in the past two decades. The NES is usually coupled with linear structures by various kinds of nonlinear coupling elements which alters the global dynamics of associated structure-NES systems to be dominated by the NES nonlinear action. Accordingly, optimum passive targeted energy transfer (TET) can be achieved by optimized NESs in a broadband frequency-energy manner [1].

NESs can be categorized according to the nature of their coupling restoring force with the associated structures. Smooth NESs include Type I NES, which incorporates a purely non-negative cubic restoring stiffness realized by transversely 
coupled linear springs [1-7], Type II NES, which includes an additional nonlinear damping element, and Type III NES, which includes an additional nonlinearly coupled mass [1-7]. Other types of smooth NESs includes the magnetic NES which is realized by a nonlinear magnetic repulsive force [8-10] and the rotary NES which is realized by inertial coupling between the NES mass and the primary structure $[11-14]$

NESs with non-smooth nonlinearities are typically realized by adding a clearance zone to separate two regions of different stiffness values in the coupling force. The piecewise linear vibration absorber for a system subjected to a narrow band harmonic loading was firstly used in [15]. In recent studies, NESs with non-smooth nonlinearities have been found to perform effective TET for broadband frequency energy ranges [2-3, 16-17]. The non-smooth stiffness nonlinearities were employed in two NESs coupled with two DOF linear primary system in [18]. The high efficiency and rapid vibration mitigation of the non-smooth stiffness NESs ignited more interest for further investigations. As a result, plenty of works studied nonsmooth NESs for implementation in TET because they become of particular interest for rapid suppression of shock and seismic excitations. A soft primary suspension and a relatively damped secondary suspension were investigated to produce a piecewise isolator that prevents the system from a high relative displacement in low frequency/high amplitude excitation [19]. A slider with two stop block was added to the piecewise NES to enable tuneable stiffness which resulted in optimum vibration absorption range [20]. A piecewise linear stiffness NES was employed in [21] where it was found effective for vibration attenuation, particularly for moderate excitation forces. This piecewise NES design was further modified in [22] by incorporating a negative coupling stiffness within the clearance zone. A 
significant improvement in the proposed piecewise NESs performance was achieved compared with other types of existing NESs. Another design modification is proposed in [23] in which the NES included negative stiffness, realized by permanent magnets, and positive piecewise linear stiffness, realized by a combination of elastic beams. The proposed system demonstrated efficient vibration suppression capability under transient and steady state excitations. A nonsmooth absorber with both piecewise stiffness and piecewise damping also showed strong vibration absorption of the steady-state dynamics of the integrated system [24]. Given the enhanced and promising performance of the piecewise NES, it was employed for vibration mitigation in rotor systems [25-26] and for energy harvesting purposes in [27-29].

To understand the nonlinear energy transfer from the linear primary structure to the NES attachment, the frequency-energy plots (FEPs) were well-investigated for several structure-NES systems. The first set of FEP studies has considered the Type I NES in which an essentially nonlinear cubic stiffness is incorporated [1-7]. Several backbone curves of nonlinear normal modes (NNMs) were generated in the FEP and analysed for different kinds of periodic motions of the structure-NES systems. In addition, different kinds of harmonic and subharmonic nonlinear periodic motions of the NNMs at the backbones and their corresponding bifurcated subharmonic tongues have been observed and analysed. The related studies of Type I NES have also employed analytical techniques for determining the fundamental backbone curves and their associated periodic orbits. However, the numerical continuation techniques in [30-32] which have been employed in [1-7, 33-36] to generate the FEPs of structure-NES systems with Type I NES were found to be providing sufficient understanding to the underlying nonlinear dynamical 
behaviour of the considered systems. Imposed wavelet transform spectrums of the frequency content in the structure-NES response on the obtained FEPs have further revealed that the TET takes place through cascades of resonance captures between the NES and the associated structure at different backbone curves and subharmonic oscillation tongues. The FEP backbone curves of the rotating NES (Type IV) were also generated in [13-14] to study the resonance capture between the rotating NES mass and the associated linear primary structure. Interesting nonlinear dynamical behaviour was observed on the backbone curves of the FEPs where different kinds of harmonic and subharmonic periodic motions were revealed. Furthermore, in the symmetric vibro-impact NES that is attached to a linear structure, several kinds of periodic orbits on the NNM branches at the FEP were obtained analytically and numerically of the considered system in [37].

Several alternative methods for understanding the frequency-energy behavior of the nonlinear dynamical systems were investigated. Firstly, a frequency versus nonlinear energy content (FNLPs) is proposed in [38] which can be directly applied into the equations of motion where numerical simulation is not required a priori. This proposed method was used in [39] to reveal the underlying nonlinear dynamical behaviour of the modal damping content of some linear systems attached to NES. Secondly, a characteristic nonlinear system identification method was proposed and implemented in [40] to identify the dynamics of local nonlinear attachments with clearance nonlinearities. The method only relies on the attachment's measured transient response data, connection points, and measured mass properties. Using this method, a frequency-displacement plot was generated for a model airplane wing equipped with a piecewise NES at the trailing edge tip. Finally, a piecewise continuation method was proposed for studying the exact 
periodic orbits of a conservative two degree-of-freedom vibro-impact system with stereo-mechanical impact model by fitting the return map equations into a scheme of continuation [41].

In the above literature, three types of NESs were studied on FEPs. This is because obtaining FEPs for the other types of NESs is more complicated, time consuming and not a straight-forward task. Therefore, the underlying nonlinear dynamic behaviour of several other types of efficient NESs is rarely studied on the FEP. Examples of such NESs include the single-sided vibro-impact NESs, the bistable NESs and the symmetric and asymmetric piecewise NESs.

Here, the piecewise nonlinear energy sink in [22] is considered for FEP analysis. The numerical continuation method in [30-32] is employed to generate the fundamental backbone curves and the subharmonics tongues that bifurcate from the lowest frequency backbone curve for two considered structure-PNES systems. Therefore, the periodic orbits of different kinds of NNMs have been obtained and discussed. In addition, the imposed wavelet transform frequency spectrum into the obtained FEPs was analysed.

\section{Piecewise NES (PNES) Description}

The PNES attachment incorporates a symmetric clearance zone of $\pm z_{c}$ width about its equilibrium position in which zero stiffness and nonzero linear viscous damping are incorporated. Moreover, the clearance boundaries are coupled with the associated linear structure by linear stiffness springs. The geometric description of PNES in Fig. 1 shows that the PNES is attached to the mass $M_{1}$ of the two degreeof-freedom linear structure according to [22]. For the relative displacement 
$w=(z-v)$ between the NES mass and $M_{1}$ in the linear structure, the piecewise coupling force is expressed as

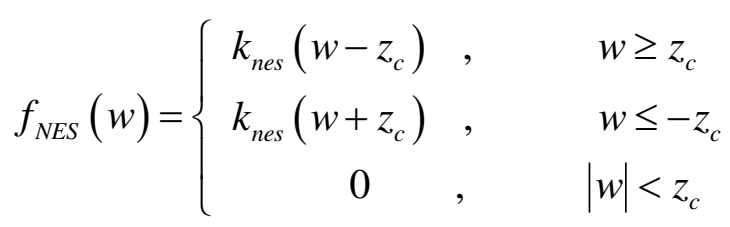

where $k_{n e s}$ is the linear stiffness coefficient and $z_{c}>0$. The vector of displacements of the structure-PNES system is expressed by $\mathbf{x}=\left[\begin{array}{lll}z & v & u\end{array}\right]^{\mathrm{T}}$ where the coupling force vector between the NES mass and the linear system is now expressed as

$$
\mathbf{F}_{N E S}(w)=\left[\begin{array}{lll}
-f_{N E S}(w) & f_{N E S}(w) & 0
\end{array}\right]^{\mathrm{T}}
$$

The equations of motion of the structure-NES system are expressed in matrix form as

$$
\mathbf{M} \mathbf{C} \&+\mathbf{K} \mathbf{x}=\mathbf{F}_{N E S}(w)
$$

where

$$
\mathbf{M}=\left[\begin{array}{ccc}
m & 0 & 0 \\
0 & M_{1} & 0 \\
0 & 0 & M_{2}
\end{array}\right], \mathbf{C}=\left[\begin{array}{ccc}
\alpha & -\alpha & 0 \\
-\alpha & \lambda_{1}+\alpha & -\lambda_{1} \\
0 & -\lambda_{1} & \lambda_{1}+\lambda_{2}
\end{array}\right] \text { and } \mathbf{K}=\left[\begin{array}{ccc}
0 & 0 & 0 \\
0 & k_{1} & -k_{1} \\
0 & -k_{1} & k_{1}+k_{2}
\end{array}\right]
$$

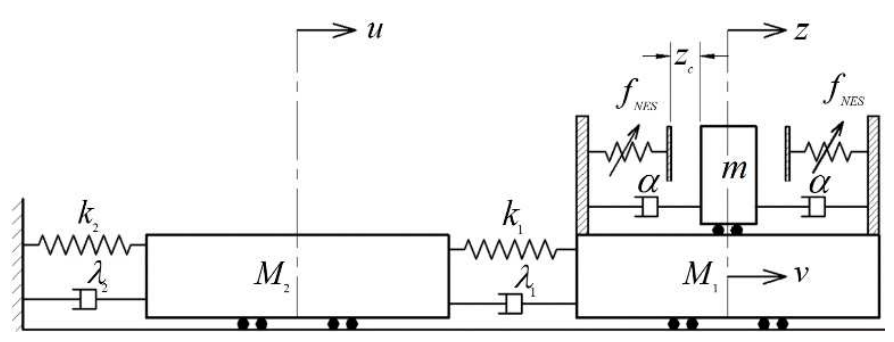

Fig. 1 Two-degree-of-freedom coupled linear oscillators attached to the PNES.

The typical parameters of the considered structure-NES system in Fig.1 are selected as $m=0.1 \mathrm{~kg}\left(m=0.05\left(M_{1}+M_{2}\right)\right), M_{1}=M_{2}=1 \mathrm{~kg}, k_{1}=k_{2}=1 \mathrm{~N} / \mathrm{m}$, 
$\lambda_{1}=\lambda_{2}=0.005 \mathrm{~N} \cdot \mathrm{s} / \mathrm{m}$ where the NES optimized parameters were obtained in [22]

as $z_{c}=0.15 \mathrm{~m}, k_{\text {nes }}=0.055 \mathrm{~N} / \mathrm{m}$.

\section{FEP using Numerical Continuation}

To obtain the FEP of the structure-PNES system using the continuation method in [30-32], the free-response of the Hamiltonian equations of motion of the system is obtained at zero damping content. Therefore, the first backbone curve of the lowest nonlinear frequency content in the FEP is obtained as shown in Fig. 2. The branches of the backbone and the bifurcated subharmonic tongues are named according to the ratio of the frequency content in the linear system to the frequency content of the NES mass response where $\mathrm{S}$ denotes to symmetric NNM periodic motion. The symmetric NNM passes through the origin with + or - slope in the NNM configuration space. For example, S13+indicates to symmetric NNM at the associated branch that passes through the origin in the configuration space with a positive slope where the oscillation frequency in the linear structure is three times that of the NES mass. Like the system with cubic stiffness NES in [1-7], the plot shows several internal resonance branches and subharmonic tongues on the backbone in Fig. 2 at which symmetric NNMs periodic motions take place. Unlike the NES with cubic stiffness, unsymmetrical NNM branches are not captured on this obtained backbone. The S11-shown in the figure interacts with S11+ backbone where the 1:1 antiphase periodic resonance motion takes place. This S11- branch which is represented in the figure by gray-colored dotted-line stands for the linear system lowest frequency. 


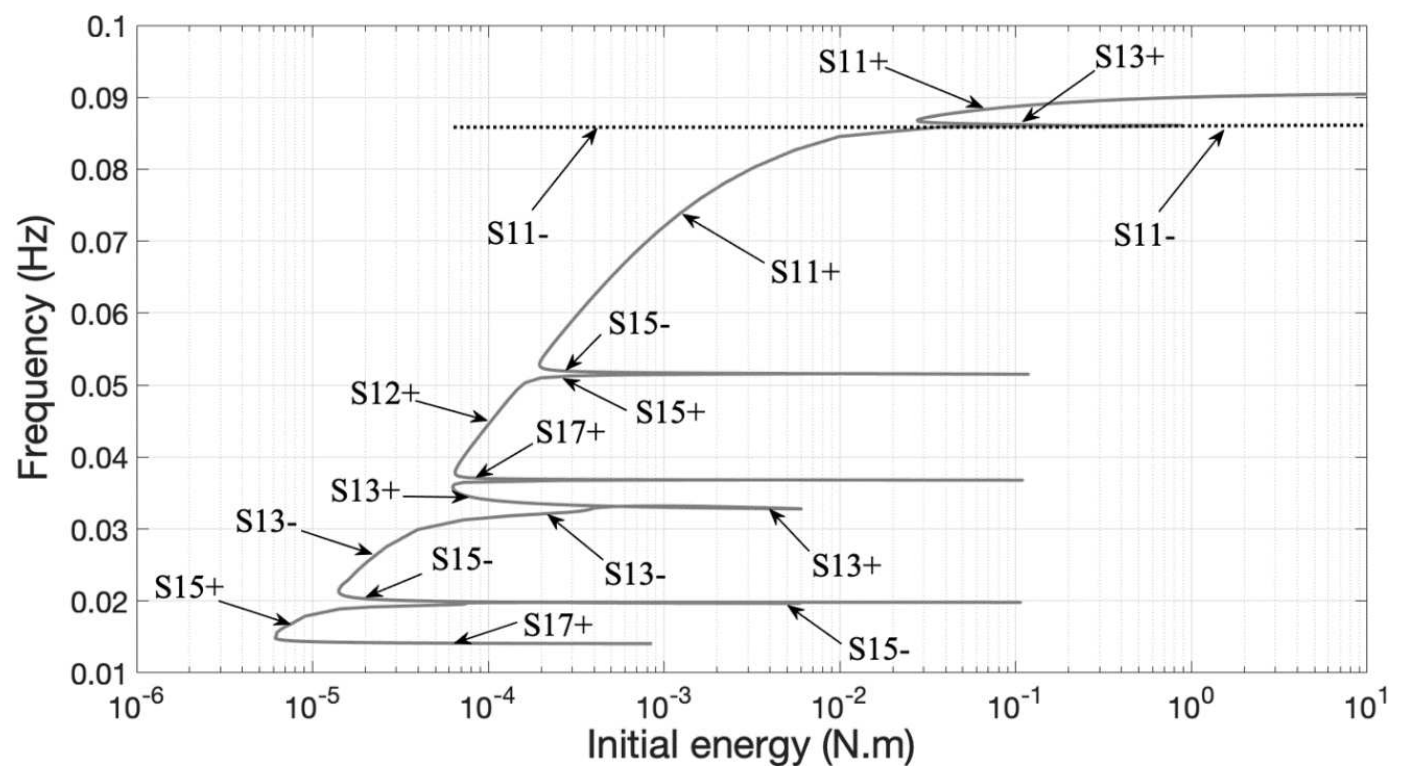

Fig 2. The lowest frequency backbone curve and its bifurcated subharmonic branches of symmetric in phase and anti-phase periodic motions on the NNMs.

The remaining high frequency backbones of S11-NNM oscillations are shown in Fig. 3. These backbone curves represent the antiphase periodic motion of $1: 1$ resonance frequency ratio within a narrow range of frequency in the $y$-axis as shown in the figure. 

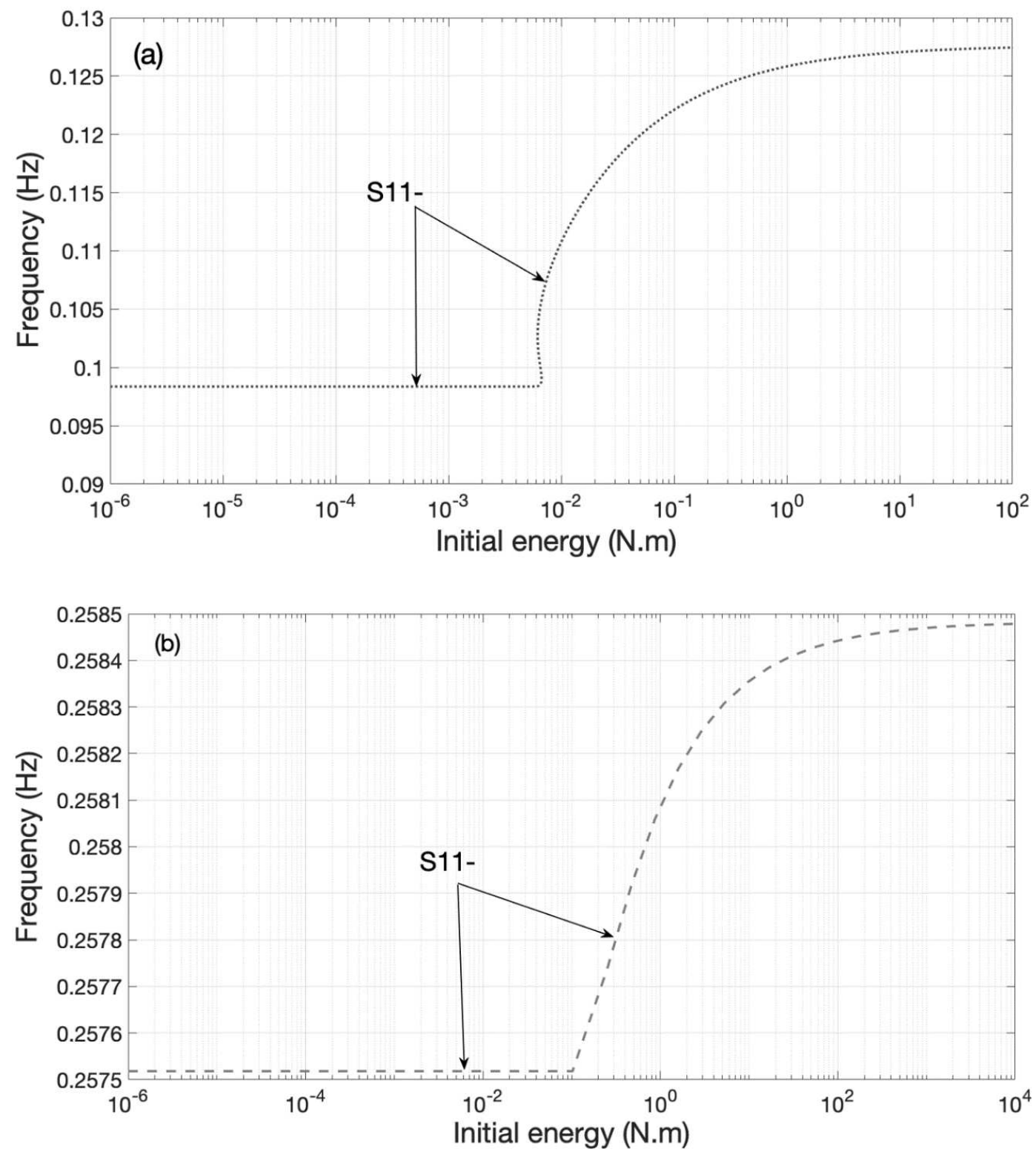

Fig 3. The antiphase intermediate frequency backbone curve in (a) and the higher frequency antiphase backbone curve in (b) of the periodic motions on the NNMs.

The obtained three backbone curves and the associated internal resonance subharmonic tongues of the lowest frequency backbone are collected together in the FEP plot in Fig. 4. Several data points have been selected on the FEP backbone curves and the subharmonic branches as shown in the figure to show their related NNMs, periodic response and the associated nonlinear force content. 


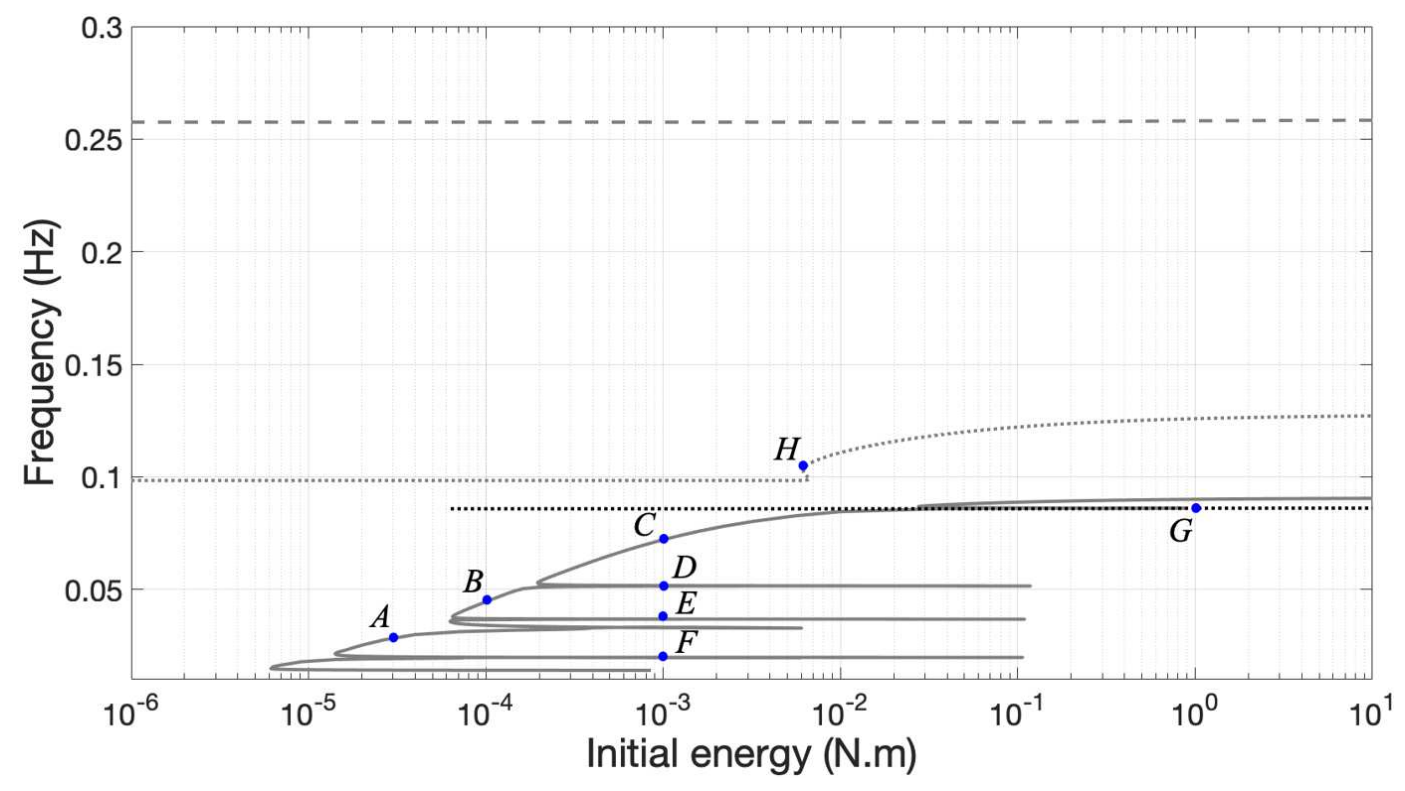

Fig 4. The three obtained backbone curves of the FEP of the structure-PNES system and their associated internal resonance subharmonic branches.

In Fig. 5, the NNM, the related periodic time response of the NES mass and the associated floor in the linear structure and the corresponding nonlinear NES coupling force are plotted for the data points $A, B$ and $C$ that are shown in Fig. 4. For the data point $A$, the NNM passes through the origin with negative slope where this NNM is located at the S13-branch of the backbone as previously shown in Fig. 1. Therefore a 1:3 resonance takes place between the NES mass and the response of the linear structure. The data point $B$ is located at $\mathrm{S} 12+$ branch of the backbone where its NNM passes through the origin with positive slope. For this data point a 1:2 resonance takes place between the NES mass and the linear structure. The 1:1 resonance between the NES mass and the structure takes place at the $\mathrm{S} 11+$ branch where the data point $C$ is selected. In all cases in Fig. 5, the nonlinear piecewise coupling force is effective in generating such strong nonlinear impact on the structure-PNES periodic response and the related NNMs. 

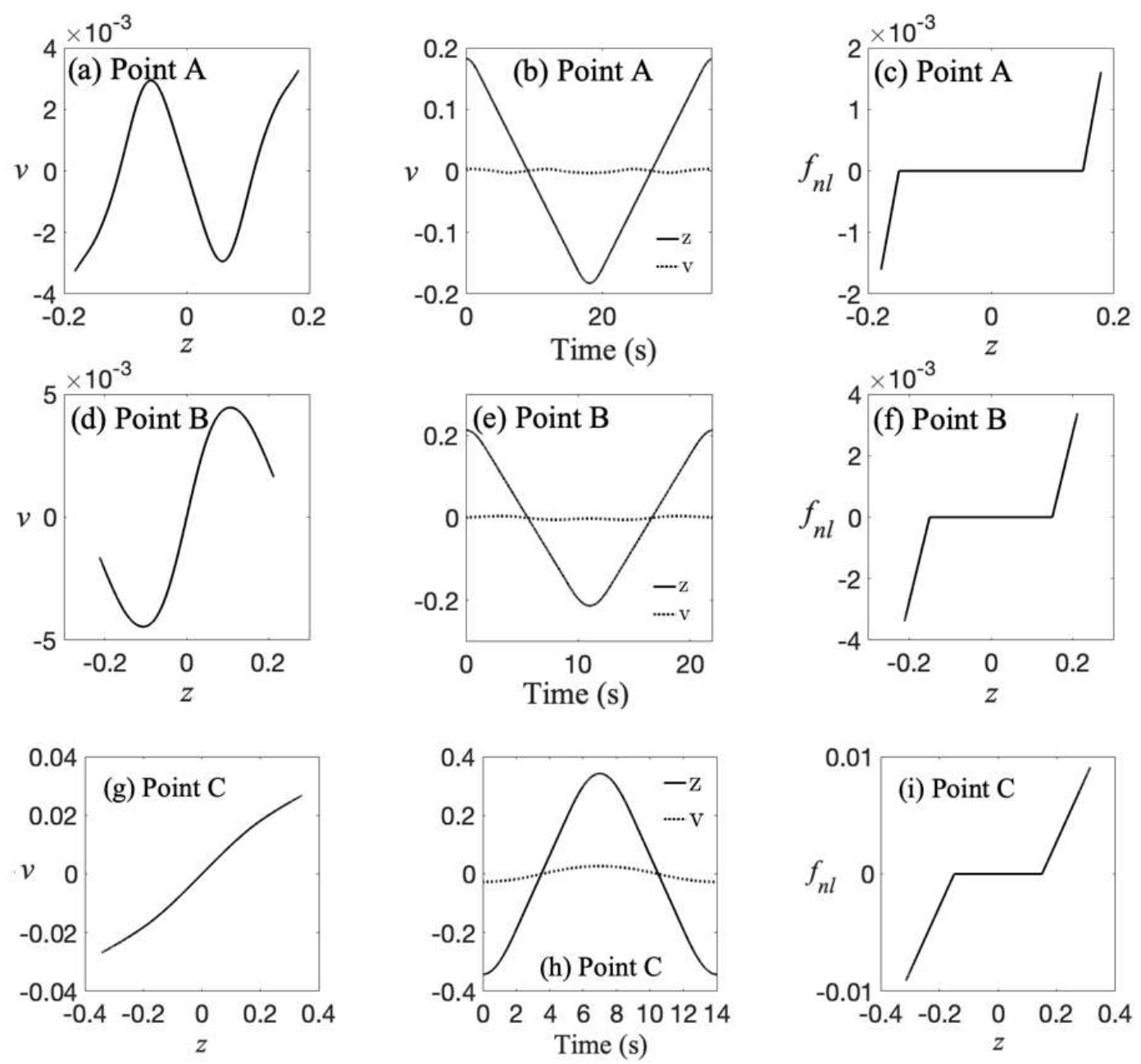

Fig 5. The NNMs in (a), (d) and (g), their corresponding response of the structure-PNES system in (b), (e) and (h), and the corresponding nonlinear NES coupling forces in (c), (f) and (i) for the data points $A, B$ and $C$ in the FEP plot in Fig. 4.

Another set of data points $(D, E$ and $F)$ is selected at the backbone subharmonic tongues at which periodic NNM motions exist. The results at these data points of NNM, periodic response and the nonlinear NES coupling force are shown in Fig. 6. These points, are located at $\mathrm{S} 15-, \mathrm{S} 17+$ and $\mathrm{S} 15+$, respectively. It is observed that for these points, the oscillation is strongly nonlinear as shown from the corresponding plots of the nonlinear coupling NES force. 

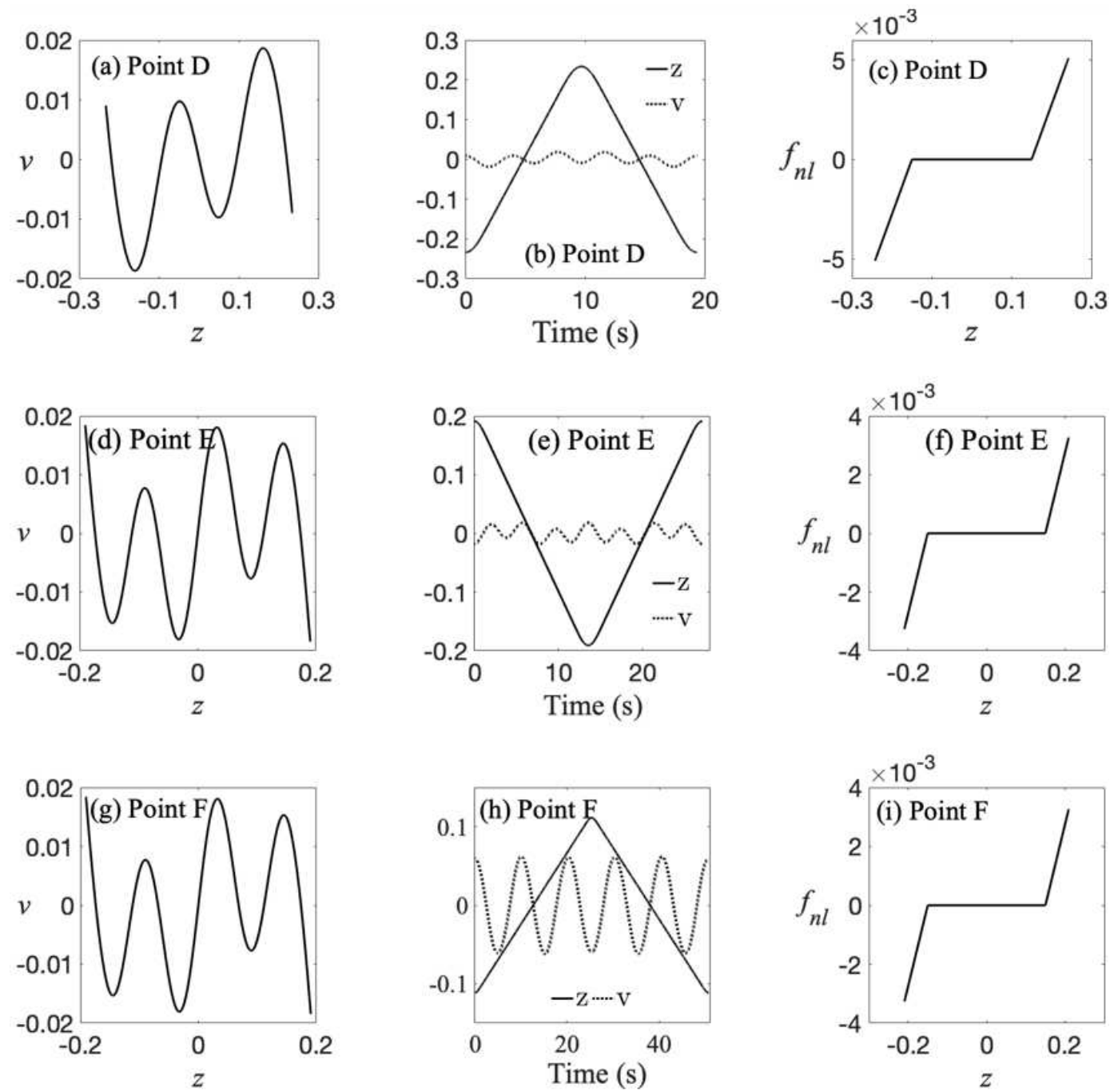

Fig 6. The NNMs in (a), (d) and (g), their corresponding response of the structure-PNES system in (b), (e) and (h), and the corresponding nonlinear NES coupling forces in (c), (f) and (i) for the data points $D, E$ and $F$ in the FEP plot in Fig. 4.

For the antiphase NNM periodic motion at the S11- backbones, two data points are selected at the locations $G$ and $H$ in the FEP. The obtained NNMs, the periodic responses and the nonlinear coupling forces of these points are plotted in Fig. 7 where both NNMs pass through the origin with negative slope and the NES mass and the linear structure exhibits a 1:1 antiphase periodic motion on these backbones. 

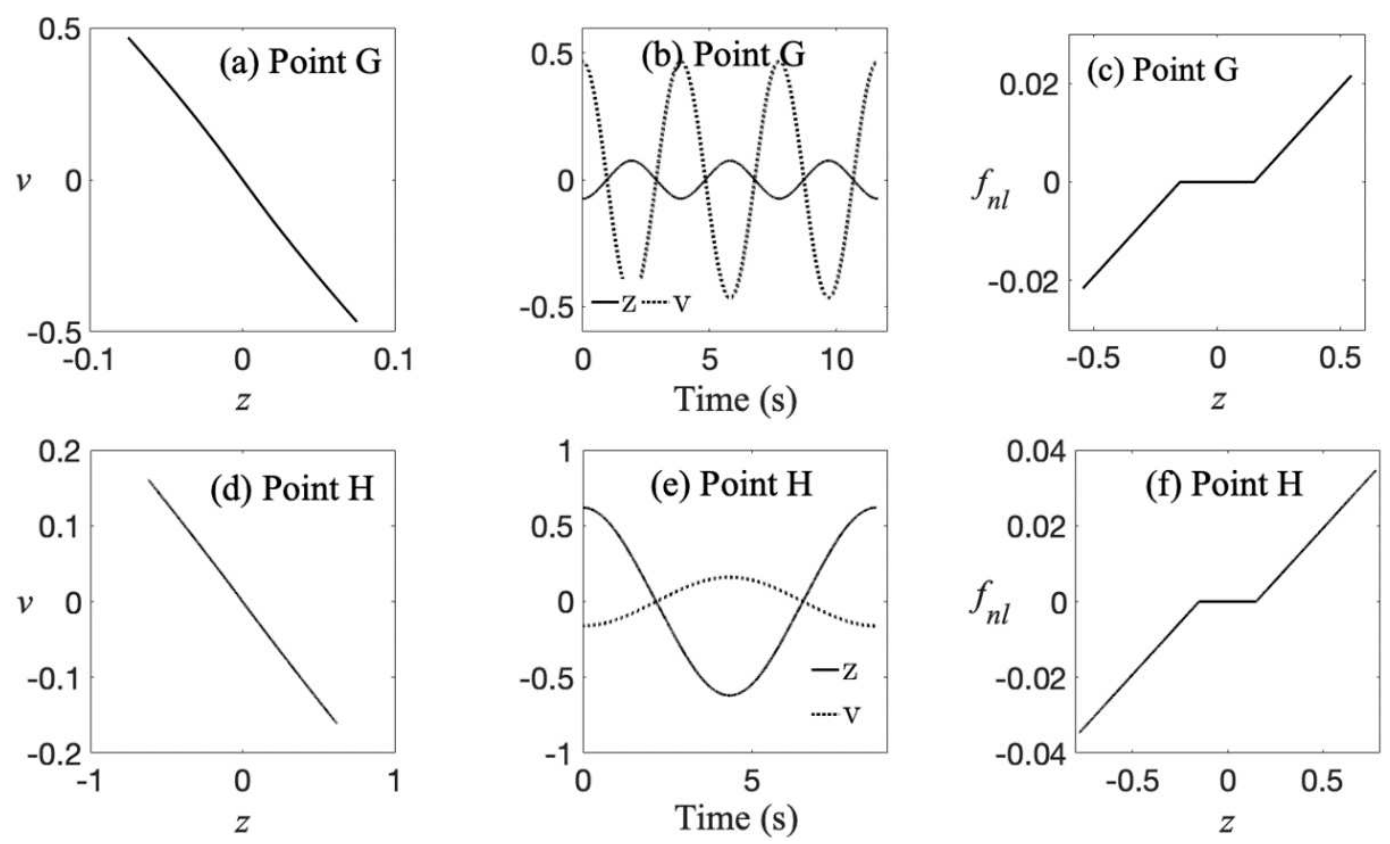

Fig 7. The antiphase NNMs in (a) and (d), their corresponding response of the structure-PNES system in (b) and (e), and the corresponding nonlinear NES coupling forces in (c) and ( $f$ ) for the data points $\mathrm{G}$ and $\mathrm{H}$ in the FEP plot in Fig. 2.

The wavelet transform spectrum for the damped response based on the relative displacement between the PNES and the linear structure is investigated here for $\lambda_{1}=\lambda_{2}=0.005 \mathrm{~N} \cdot \mathrm{s} / \mathrm{m}$ of the linear structure and $\alpha=0.001 \mathrm{~N} \cdot \mathrm{s} / \mathrm{m}$ of the PNES. The results are shown in Fig. 8 for low and high initial velocities induced equally to the masses of the linear structure at zero initial displacements. A strong resonance capture is observed in Fig. 8a with two backbone curves. In addition, a cascade of resonance captures with the internal resonance subharmonic tongues of the lowest frequency backbone is also observed. In Fig. 8b, at higher initial velocities of the structure, strong resonance capture immediately takes place with the two backbone curves as shown where the resonance capture with decaying frequency continues along the low frequency backbone. These imposed wavelet observations show the strongly nonlinear dynamical behaviour of the PNES on the FEP where the TET is achieved by several resonance captures between the PNES mass and the linear structure at different backbone curves and subharmonic tongues. 

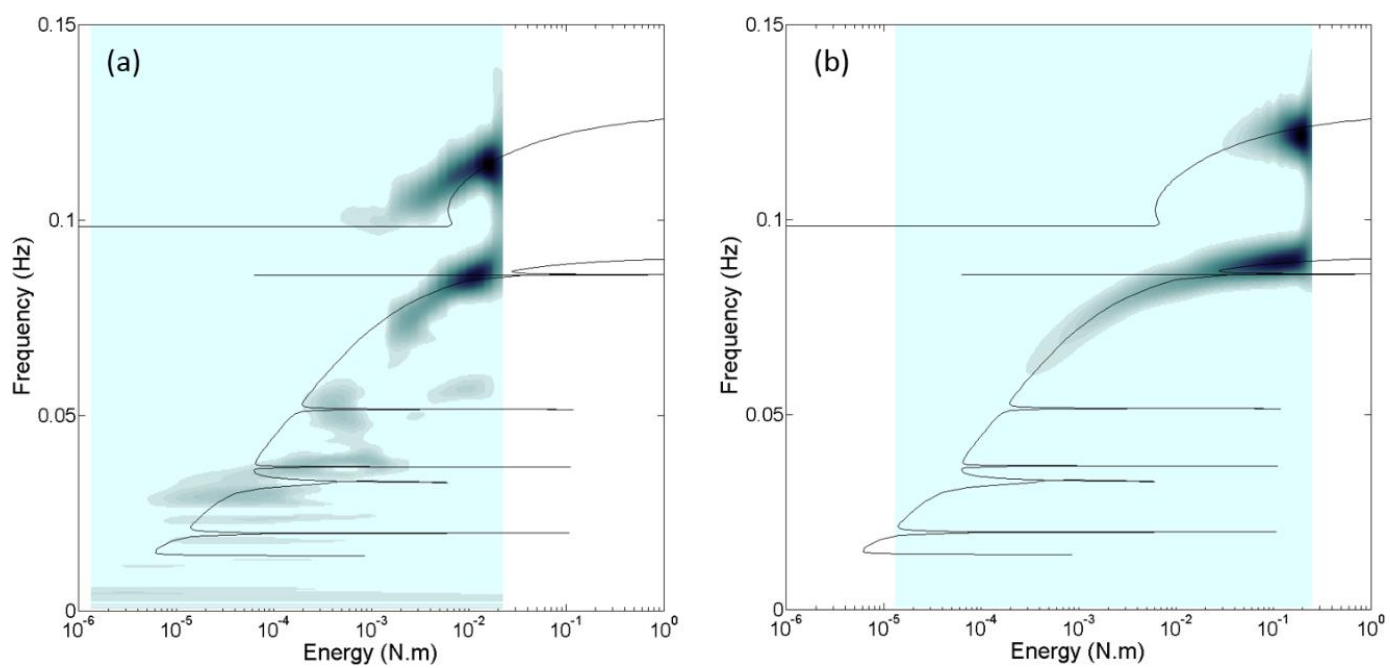

Fig. 8 Imposed wavelet transform frequency spectrum on the FEP of the damped response of the structure-PNES system in (a) at $u(0)=v(0)=0.15 \mathrm{~m} / \mathrm{s}$ and in (b) at $u(0)=v(0)=0.5 \mathrm{~m} / \mathrm{s}$.

\section{FEP for Physical Two-Story Structure-PNES system}

The identified physical parameters of the two-story lab structure in [11] are considered here for generating the FEP plot and investigating the dynamics of the optimized PNES with this structure. The optimized parameters of the PNES attachment to this structure are obtained as $m=2.8 \mathrm{~kg}, \quad z_{c}=0.02 \mathrm{~m}$ and $k_{\text {nes }}=800 \mathrm{~N} / \mathrm{m}$. Accordingly the structure-PNES system mass, stiffness and damping matrices are, respectively, rewritten as

$$
\mathbf{M}=\left[\begin{array}{ccc}
2.8 & 0 & 0 \\
0 & 24.3 & 0 \\
0 & 0 & 24.3
\end{array}\right], \mathbf{C}=\left[\begin{array}{ccc}
3.4 & -3.4 & 0 \\
-3.4 & 4.368 & -0.295 \\
0 & -0.295 & 1.263
\end{array}\right] \text { and } \mathbf{K}=\left[\begin{array}{ccc}
0 & 0 & 0 \\
0 & 8220 & -8220 \\
0 & -8220 & 15440
\end{array}\right]
$$

The optimized PNES with this structure was found dissipating $96 \%$ of the input energy induced into the structure by impulsive loading at $\iota \&(0)=\operatorname{lo}(2)=0.24 \mathrm{~m} / \mathrm{s}$. To verify that this high energy dissipation mainly takes place by the nonlinear action of the PNES, the FEP backbones curves and their associated internal resonance subharmonic tongues are generated as shown in Fig. 9. Unlike the 
Hamiltonian case shown in Fig. 4, unsymmetrical subharmonic NNM branches are now captured on the FEP.

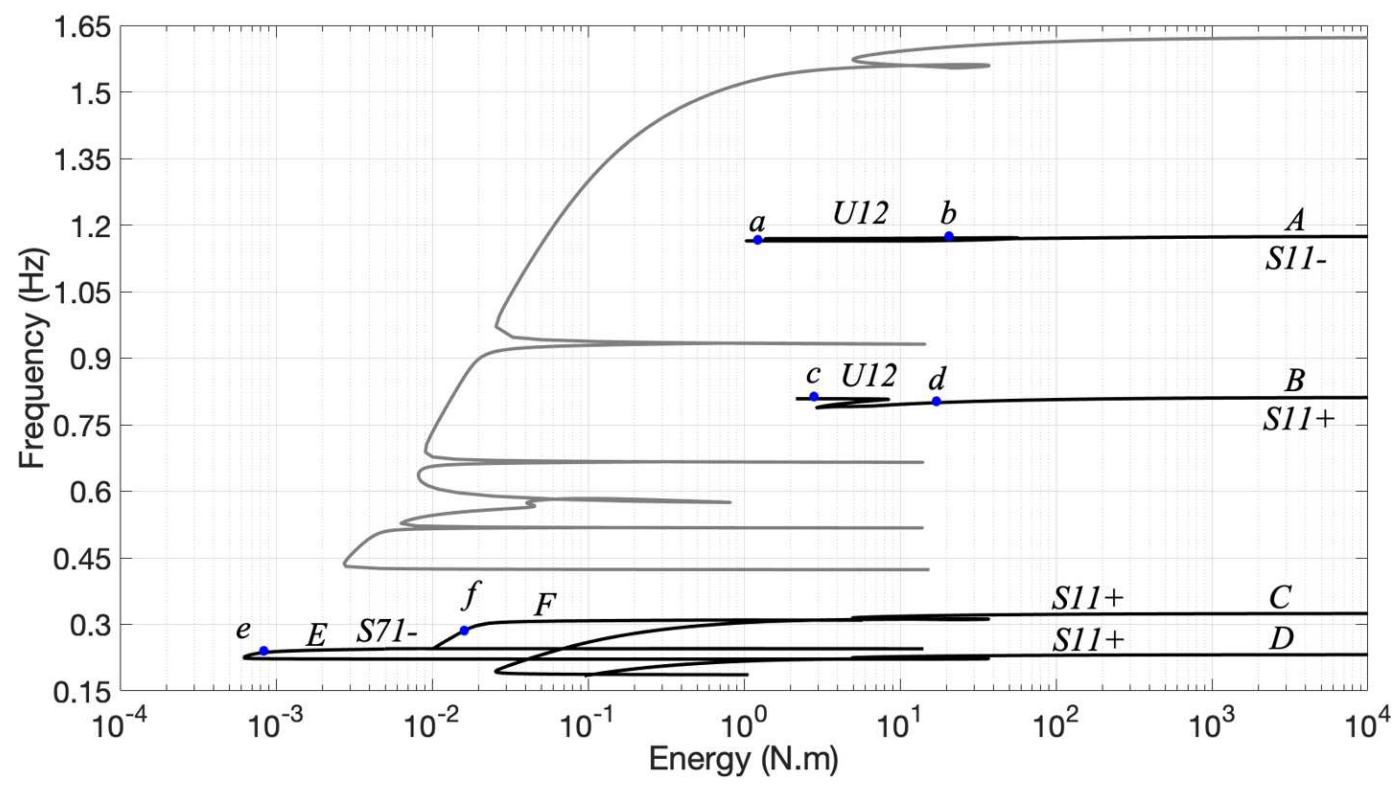

Fig. 9 The lowest frequency backbone curve and its associated subharmonic branches and tongues of symmetrical and unsymmetrical NNMs periodic motions.

Several data points have been selected here on the FEP subharmonic branches as shown in Fig. 9 to obtain their related NNMs, periodic response and their nonlinear force content as shown in Figs. 10-12. The periodic motion of the system is demonstrated through NNMs, periodic responses and piecewise nonlinear forces in Fig. 10 of the data points $a$ and $b$ and in Fig. 11 of data points $c$ and $d$. The unsymmetrical NNMs of 2:1 resonance appeared at points $a, b$, and $c$ which are all selected at U12 tongues on $A$ and $B$ subharmonic branches. However, at subharmonic branch $B$ the data point $d$ shows symmetrical 1:1 resonance on the NNM.

For data point $e$ which is selected at low frequency content subharmonic branch, a symmetric antiphase 1:7 resonance takes place as shown in Fig. 12. This indicates to the highly nonlinear dynamical behaviour of the structure-PNES system. However, the response of data point $f$ is found to be dominated by the in phase $1: 1$ 
resonance as shown in Fig. 12d-12f. For all cases in Figs. 10-12, the strong nonlinear effect of the PNES is observed in the NNMs and the nonlinear piecewise coupling force plots.
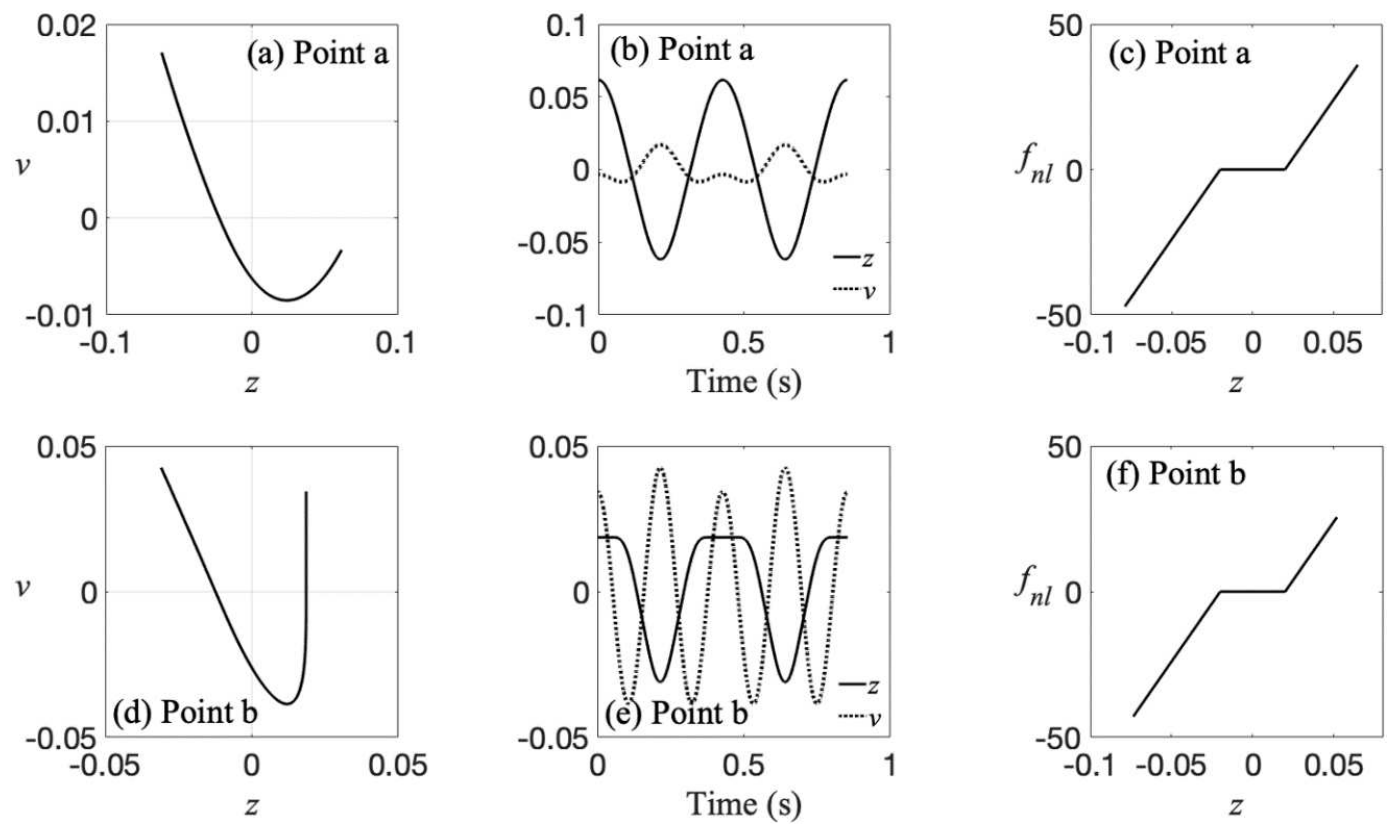

Fig 10 The antiphase NNMs in (a) and (d), their corresponding response of the structure-PNES system in (b) and (e), and the corresponding nonlinear NES coupling forces in (c) and ( $f$ ) for the data points $a$ and $b$ in the FEP plot in Fig. 9.
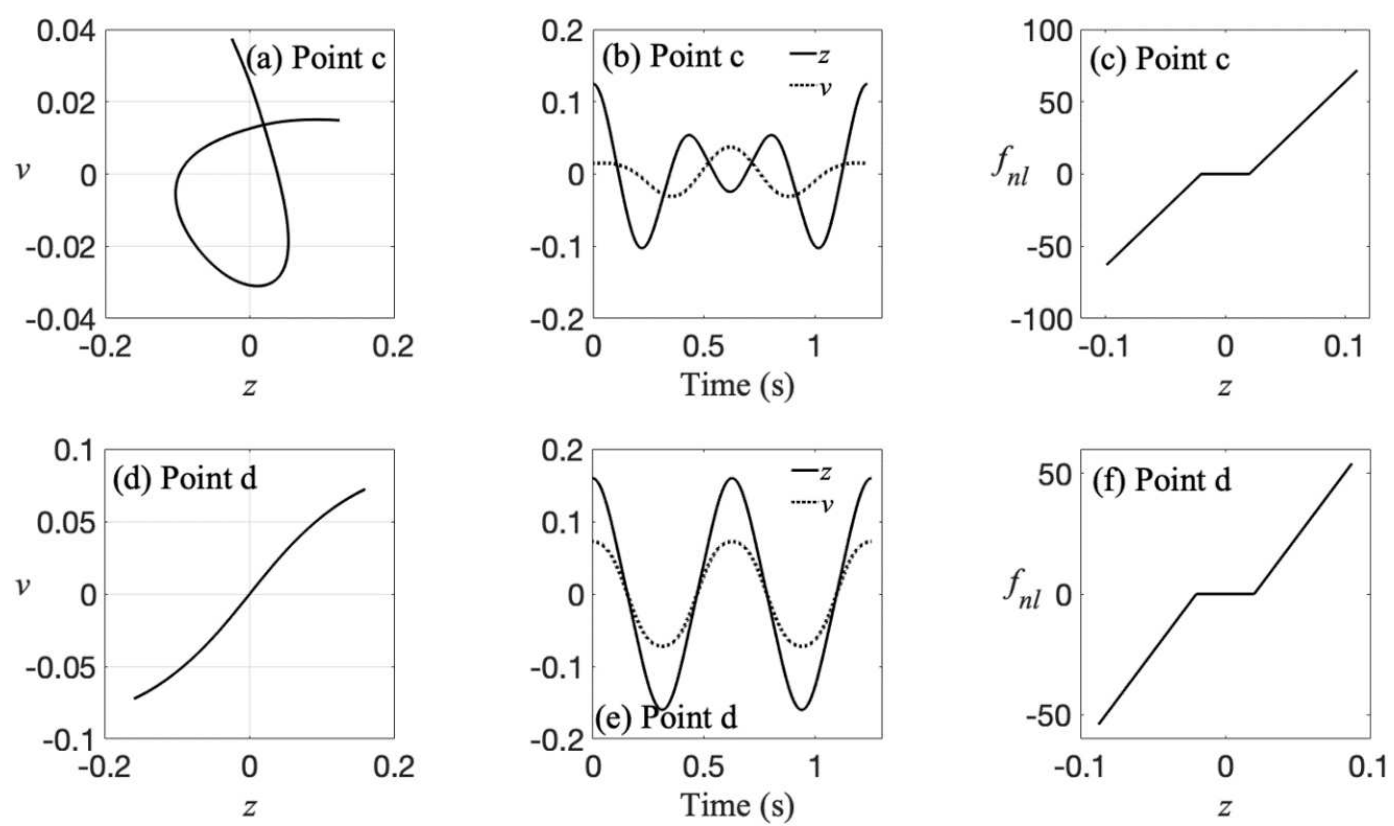

Fig 11 The antiphase NNMs in (a) and (d), their corresponding response of the structure-PNES system in (b) and (e), and the corresponding nonlinear NES coupling forces in (c) and (f) for the data points $c$ and $d$ in the FEP plot in Fig. 9. 

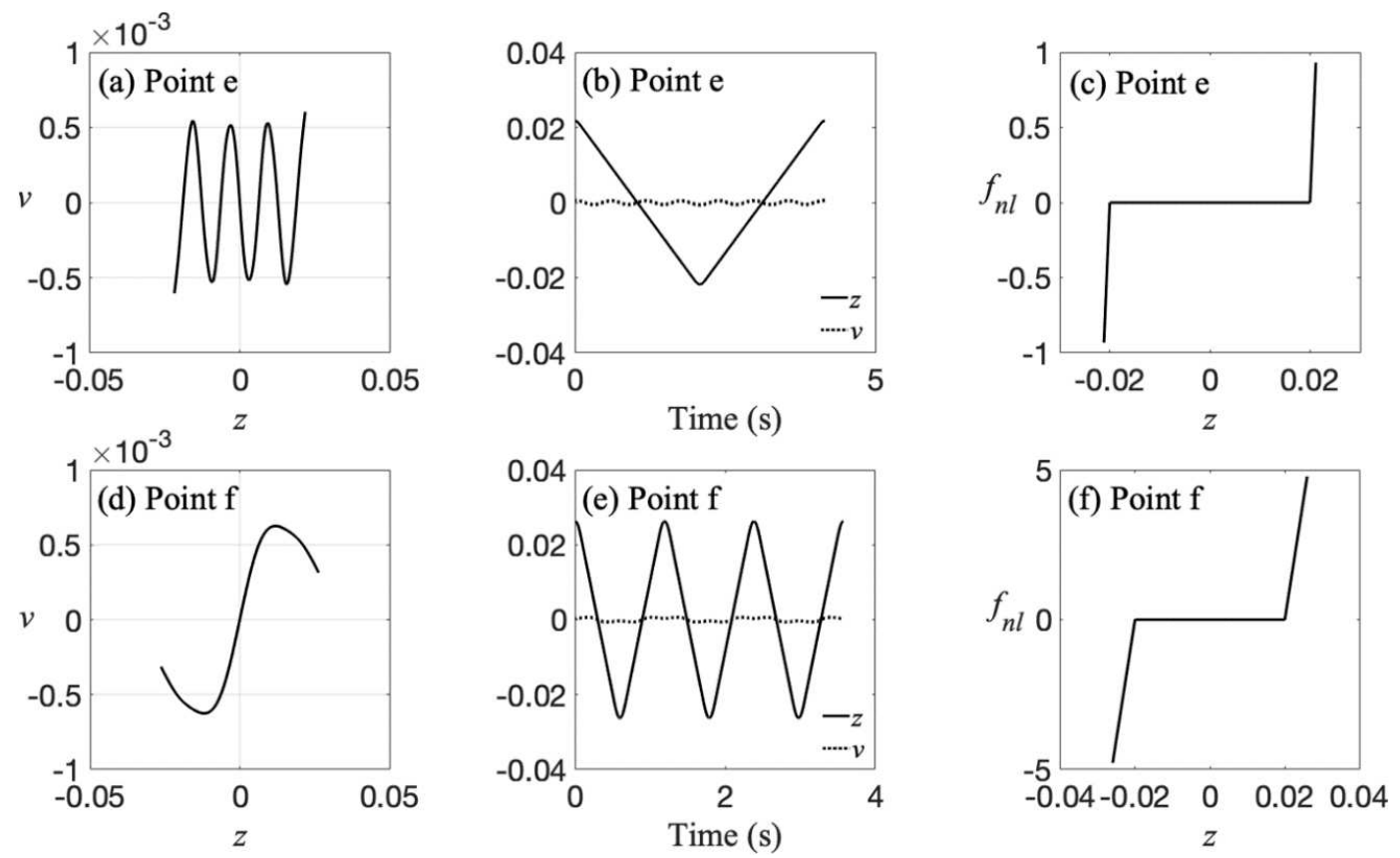

Fig 12 The antiphase NNMs in (a) and (d), their corresponding response of the structure-PNES system in (b) and (e), and the corresponding nonlinear NES coupling forces in (c) and ( $f$ ) for the data points $e$ and $f$ in the FEP plot in Fig. 9.

The detailed FEP is now shown in Fig. 13 which incorporates all aforementioned obtained backbones and subharmonic branches. Therefore, this FEP plot is employed with wavelet transform frequency spectrum analysis for the damped response of the relative displacement between the PNES and the linear structure as shown in Fig. 14. For varying identical nonzero initial velocities (low, intermediate and high initial velocities) induced into the linear structure, the figure shows strong resonance captures with two backbone curves for all considered initial velocities besides to cascades of resonance captures with the low frequency subharmonic branches. The imposed wavelet findings indicate to that, the PNES is engaged in several resonance captures with the linear structure by its nonlinear action. Accordingly, rapid TET has been achieved which renders this NES to be efficient to function as passive vibration absorber. This demonstrates that the TET 
is strongly dominated by the nonlinear action of the PNES rather than the effect of linear coupling stiffness and damping elements.

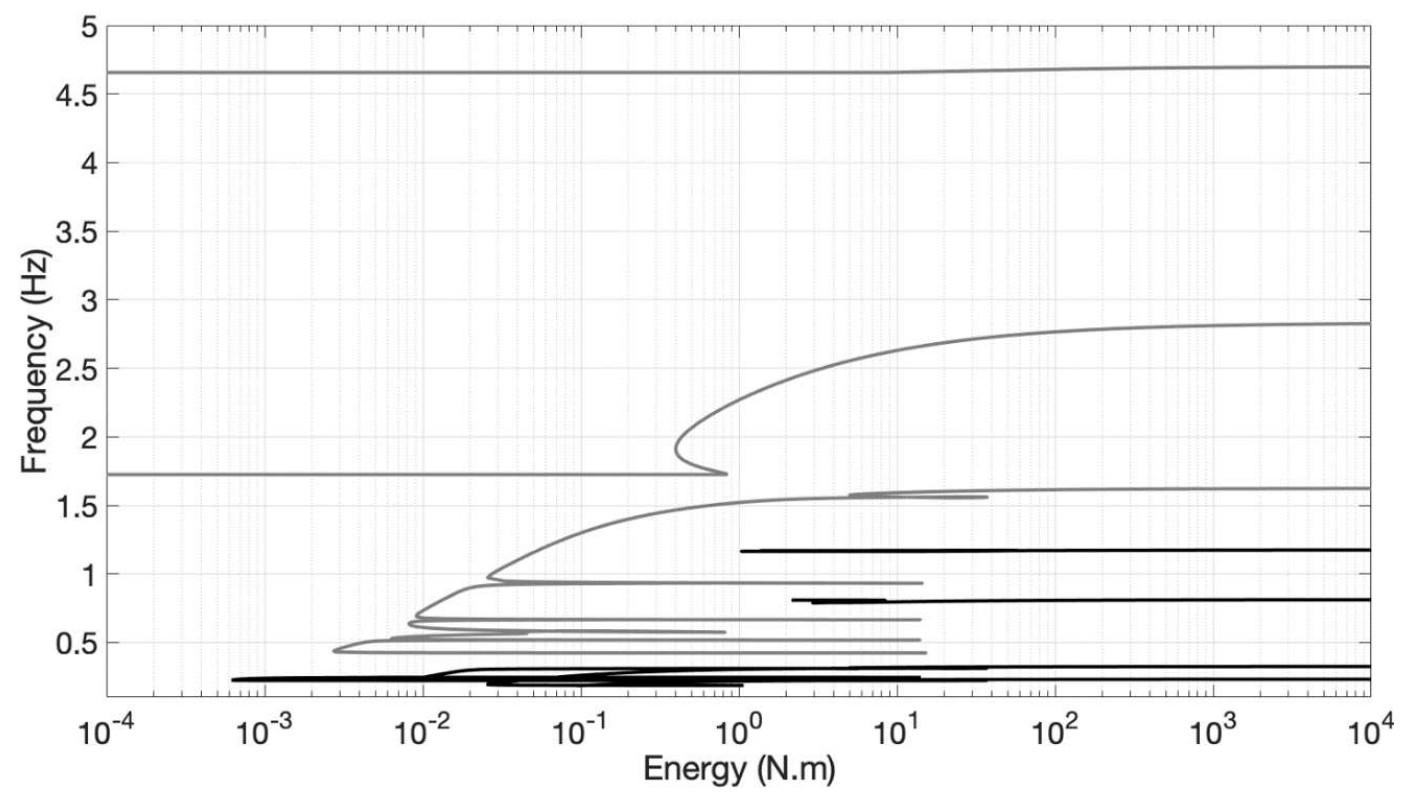

Fig 13 The three obtained backbone curves of the FEP of the structure-PNES system and the associated subharmonic branches of the NNMs periodic motion. 

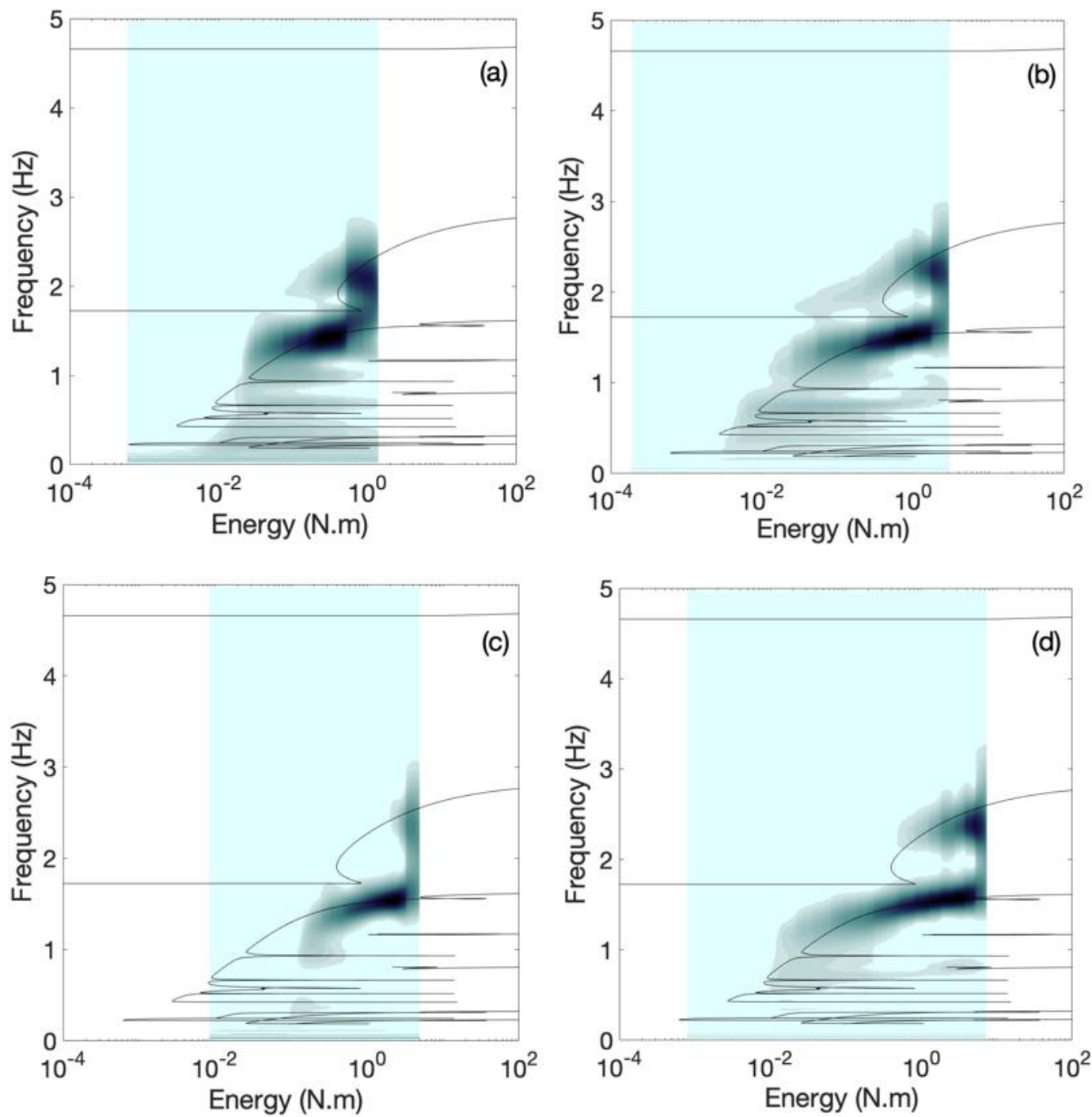

Fig. 14 Imposed wavelet transform frequency spectrum on the FEP of the damped response of the structure-PNES system in (a) at $ı \& 0)=\& \&(0)=0.025 \mathrm{~m} / \mathrm{s}$, (b) at $ı \&(0)=1 \&(2)=0.035 \mathrm{~m} / \mathrm{s}$, (c) at $\iota \&(0)=\& \& 0)=0.045 \mathrm{~m} / \mathrm{s}$ and $($ b) at $\iota \&(0)=1 \&(0)=0.055 \mathrm{~m} / \mathrm{s}$.

\section{Concluding remarks}

In this study, the underlying nonlinear dynamical behaviour of coupled piecewise nonlinear energy sink (PNES) with two-degree-of-freedom linear dynamical structures is investigated on the frequency energy plot (FEP). Accordingly, the three fundamental backbone curves of the low, intermediate and high frequency content in the FEPs have been generated by numerical simulation and NNM continuation methods. In addition, several bifurcated subharmonic branches (tongues) at low frequency backbone curve have been also obtained. 
Therefore, coupling the PNES with linear structures has shown significant nonlinear dynamical behaviour on the FEP, especially at the lowest and intermediate frequency backbone curves. Furthermore, imposing wavelet transform frequency spectrum of the structure-PNES response on the FEP has shown strong cascades of resonance captures and clear nonlinear dynamical interaction between the PNES and the linear structure at the backbone curves and subharmonic frequency branches. The findings verify that the energy transfer and dissipation by the PNES is strongly dominated by its nonlinear action in the considered systems rather than the linear coupling elements in the considered structure-PNES system.

\section{Conflict of Interest}

The authors declare that they have no conflict of interest.

\section{References}

[1] Vakakis, A. F., Gendelman, O. V., Bergman, L. A., McFarland, D. M., Kerschen, G., Lee, Y. S.: Nonlinear targeted energy transfer in mechanical and structural systems, Vol. 156. Springer Science \& Business Media (2008).

[2] Vakakis, A.F., and Gendelman, O.: Energy pumping in nonlinear mechanical oscillators: part II resonance capture. J. Appl. Mech. 68(1), 42-48 (2001). https://doi.org/10.1115/1.1345525

[3] Gendelman, O., Manevitch, L., Vakakis, A.F., Mcloskey, R.: Energy pumping in nonlinear mechanical oscillators: Part I dynamics of the underlying hamiltonian systems. J. Appl. Mech. 68(1), 34-41 (2001). https://doi.org/10.1115/1.1345524

[4] Vakakis, A.F.: Shock isolation through the use of nonlinear energy sinks. J. Vib. and Cont. 9(1), pp. 79-93 (2003). https://doi.org/10.1177/107754603030742

[5] Lee, Y.S., Kerschen, G., Vakakis, A.F., Panagopoulos, P., Bergman, L., McFarland, D.M.: Complicated dynamics of a linear oscillator with a light, essentially nonlinear attachment. Physica D 204(1-2), pp. 41-69 (2005). https://doi.org/10.1016/j.physd.2005.03.014

[6] Gourdon, E., Alexander, N. A., Taylor, C.A., Lamarque, C.-H., Pernot, S.: Nonlinear energy pumping under transient forcing with strongly nonlinear coupling: Theoretical 
and experimental results. J. Sound Vib. 300(3-5), 522-551 (2007).

https://doi.org/10.1016/j.jsv.2006.06.074

[7] Quinn, D.D., Gendelman, O., Kerschen, G., Sapsis, T.P., Bergman, L.A., Vakakis, A.F.:

Efficiency of targeted energy transfers in coupled nonlinear oscillators associated with 1 : 1 resonance captures: Part I. J. Sound Vib. 311(3-5), 1228-1248 (2008).

https://doi.org/10.1016/j.jsv.2007.10.026

[8] Benacchio, S., Malher, A., Boisson, J., Touzé, C.: Design of a magnetic vibration absorber with tunable stiffnesses. Nonlinear Dyn. 85, 893-911 (2016).

https://doi.org/10.1007/s11071-016-2731-3

[9] Kremer, D., Liu, K.: A nonlinear energy sink with an energy harvester: Transient responses, J. Sound Vib. 333(20), 4859-4880 (2014).

https://doi.org/10.1016/j.jsv.2014.05.010

[10] Lo Feudo, S., Touzé, C., Boisson, J., Cumunel, G.: Nonlinear magnetic vibration absorber for passive control of a multi-storey structure, J. Sound Vib. 438, 33-53 (2019). https://doi.org/10.1016/j.jsv.2018.09.007

[11] Saeed, A.S., Al-Shudeifat, M.A., Vakakis, A.F.: Rotary-oscillatory nonlinear sink of robust performance. Int. J. Non-Linear Mech. 117, 103249 (2019).

https://doi.org/10.1016/j.ijnonlinmec.2019.103249

[12] Saeed, A.S., AL-Shudeifat, M.A., Cantwell, W.J., Valais, A.F.: Rotary-impact nonlinear energy sink for shock mitigation: analytical and numerical investigations. Arch. Appl. Mech. 90, 495-521 (2020). https://doi.org/10.1007/s00419-019-01622-0

[13] Sigillo, G., Gendelman, O.V., AL-Shediac, M.A., Mane itch, L.I., Volakis, A.F., Bergman, L.A.: Resonance captures and targeted energy transfers in an inertially-coupled rotational nonlinear energy sink. Nonlinear Dyn. 69, 1693-1704 (2012). https://doi.org/10.1007/s11071-012-0379-1

[14] AL-Hodeida, M.A., Wierschem, N.E., Bergman, L.A., Vakakis, A.F.: Numerical and experimental investigations of a rotating nonlinear energy sink. Meccanica 52, 763-779 (2017). https://doi.org/10.1007/s11012-016-0422-2

[15] Pun, D., Liu, Y.B.: On the Design of the Piecewise Linear Vibration Absorber. Nonlinear Dyn. 22, 393-413 (2000). https://doi.org/10.1023/A:1008309409223

[16] Gendelman, O.V.: Transition of Energy to a Nonlinear Localized Mode in a Highly Asymmetric System of Two Oscillators. Nonlinear Dyn. 25, 237-253 (2001). https://doi.org/10.1023/A:1012967003477

[17] Vakakis, A.F.: Inducing Passive Nonlinear Energy Sinks in Vibrating Systems. J. Vib. Acoustics 123(3), pp. 324-332 (2001). https://doi.org/10.1115/1.1368883

[18] Georgiadis, F., Vakakis, A.F., Mcfarland, D.M., Bergman, L.: Shock Isolation Through Passive Energy Pumping Caused By Nonsmooth Nonlinearities. Int. J. of Bifurcation \& Chaos 15(06), pp. 1989-2001 (2005). https://Doi.Org/10.1142/S0218127405013101

[19] Jazar, G.N., Mahinfalah, M., Deshpande, S.: Design of a piecewise linear vibration isolator for jump avoidance. Proceedings of the Institution of Mechanical Engineers, Part 
K: Journal of Multi-body Dynamics 221(3), pp. 441-449 (2007).

https://doi.org/10.1243/14644193JMBD56

[20] Shui, X., Wang, S.: Investigation on a mechanical vibration absorber with tunable piecewise-linear stiffness. Mech. Syst. Signal Process 100, pp. 330-343 (2018). https://doi.org/10.1016/i.ymssp.2017.05.046

[21] Yao, H., Cao, Y., Zhang, S. et al.: A novel energy sink with piecewise linear stiffness. Nonlinear Dyn. 94, 2265-2275 (2018). https://doi.org/10.1007/s11071-018-4488-3

[22] AL-Shudeifat, M.A.: Nonlinear Energy Sinks with Piecewise-Linear Nonlinearities. J. Comput. Nonlinear Dyn 14(12), pp. 124501(2019). https://doi.org/10.1115/1.4045052

[23] Yao, H., Cao, Y., Wang, Y., Wen, B.: A tri-stable nonlinear energy sink with piecewise stiffness. J. Sound Vib. 463, pp. 114971 (2019). https://doi.org/10.1016/i.jsv.2019.114971

[24] Sun, M., Hu, Wh., Liu, J. et al.: Steady-state responses of mechanical system attached to non-smooth vibration absorber with piecewise damping and stiffness. Meccanica (2021). https://doi.org/10.1007/s11012-020-01288-x

[25] Yao, H., Wang, Y., Cao, Y., Wen, B.: Multi-stable nonlinear energy sink for rotor system. Int. J. Non-Linear Mech. 118, 103273 (2020). https://doi.org/10.1016/i.ijnonlinmec.2019.103273

[26] Yao, H., Cao, Y., Ding, Z., Wen, B.: Using grounded nonlinear energy sinks to suppress lateral vibration in rotor systems. Mech. Syst. Signal Process 124, pp. 237253 (2019). https://doi.org/10.1016/i.ymssp.2019.01.054

[27] Zhang, K., Hou, Y.D., Tian, W.X., Zhang, Y.P., Su, G.H., Qiu, S.Z.: Experimental investigations on single-phase convection and two-phase flow boiling heat transfer in an inclined rod bundle. Applied Thermal Engineering 148, pp. 340-351 (2019). https://doi.org/10.1016/j.applthermaleng.2018.11.067

[28] Fan, K., Tan, Q., Liu, H., Zhang, Y., Cai, M.: Improved energy harvesting from lowfrequency small vibrations through a monostable piezoelectric energy harvester. Mech. Syst. Signal Process 117, pp. 594-608 (2019). https://doi.org/10.1016/j.ymssp.2018.08.001

[29] Zoka, H., Afsharfard, A.: Double stiffness vibration suppressor and energy harvester: An experimental study. Mech. Syst. Signal Process 121, pp. 1-13 (2019). https://doi.org/10.1016/i.ymssp.2018.11.020

[30] Kerschen, G., Peeters, M., Golinval, J.C.: Nonlinear normal modes, Part I: A useful framework for the structural dynamicist. Mech. Syst. Signal Process. 23(1), 170-194 (2009). https://doi.org/10.1016/j.ymssp.2008.04.002

[31] Peeters, M., Viguié, R., Sérandour, G., Kerschen, G., Golinval, J.C.: Nonlinear normal modes, Part II: Toward a practical computation using numerical continuation techniques. Mech. Syst. Signal Process. 23(1), 195-216 (2009). https://doi.org/10.1016/i.ymssp.2008.04.003 
[32] Renson, L., Kerschen, G., Cochelin, B.: Numerical computation of nonlinear normal modes in mechanical engineering. J. Sound Vib. 364, 177-206 (2016).

https://doi.org/10.1016/j.jsv.2015.09.033

[33] Tsakirtzis, S., Panagopoulos, P. N., Kerschen, G., Gendelman, O., Vakakis, A. F., Bergman, L. A.: Complex dynamics and targeted energy transfer in linear oscillators coupled to multi-degree-of-freedom essentially nonlinear attachments. Nonlinear Dyn. 48(3), 285318 (2007). https://doi.org/10.1007/s11071-006-9089-x

[34] Kerschen, G., Vakakis, A. F., Lee, Y. S., McFarland, D. M., Kowtko, J. J., Bergman, L. A.: Energy transfers in a system of two coupled oscillators with essential nonlinearity: 1: 1 resonance manifold and transient bridging orbits. Nonlinear Dyn. 42(3), 283-303 (2005). https://doi.org/10.1007/s11071-005-4475-3

[35] Kerschen, G., Kowtko, J. J., McFarland, D. M., Bergman, L. A., Vakakis, A. F.: Theoretical and experimental study of multimodal targeted energy transfer in a system of coupled oscillators. Nonlinear Dyn. 47(1-3), 285-309 (2007). https://doi.org/10.1007/s11071-006-9073-5

[36] Haris, A., Alevras, P., Mohammadpour, M. et al.: Design and validation of a nonlinear vibration absorber to attenuate torsional oscillations of propulsion systems. Nonlinear Dyn 100, 33-49 (2020). https://doi.org/10.1007/s11071-020-05502-z

[37] Lee, Y. S., Nucera, F., Vakakis, A.F., McFarland, D.M., Bergman, L.A.: Periodic orbits, damped transitions and targeted energy transfers in oscillators with vibro-impact attachments. Physica D: Nonlinear Phenomena 238(18), 1868-1896 (2009). https://doi.org/10.1016/i.physd.2009.06.013

[38] AL-Shudeifat, M. A.: Time-varying stiffness method for extracting the frequency-energy dependence in the nonlinear dynamical systems. Nonlinear Dyn. 89(2), 1463-1474 (2017). https://doi.org/10.1007/s11071-017-3528-8

[39] Al-Shudeifat, M. A.: Modal damping variations in nonlinear dynamical systems. Nonlinear Dyn. 93(4), 2565-2578 (2018). https://doi.org/10.1007/s11071-018-4342-7

[40] Singh, A., Moore, K.J.: Characteristic nonlinear system identification of local attachments with clearance nonlinearities. Nonlinear Dyn. 102, 1667-1684 (2020). https://doi.org/10.1007/s11071-020-06004-8

[41] Tao, H., Gibert, J.: Periodic orbits of a conservative 2-DOF vibro-impact system by piecewise continuation: bifurcations and fractals. Nonlinear Dyn. 95, 2963-2993 (2019). https://doi.org/10.1007/s11071-018-04734-4 
Figures

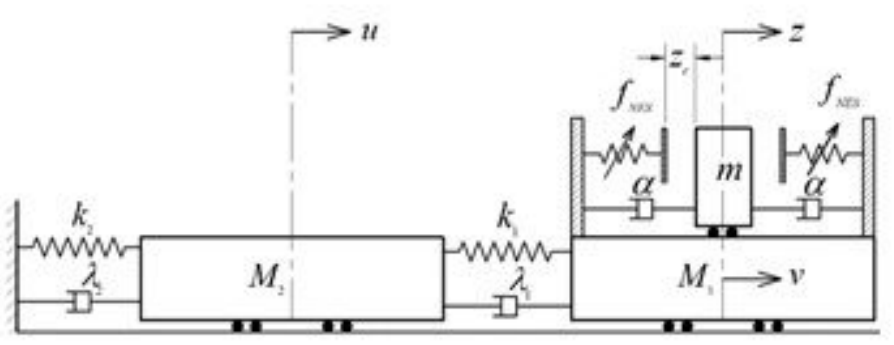

\section{Figure 1}

Two-degree-of-freedom coupled linear oscillators attached to the PNES.

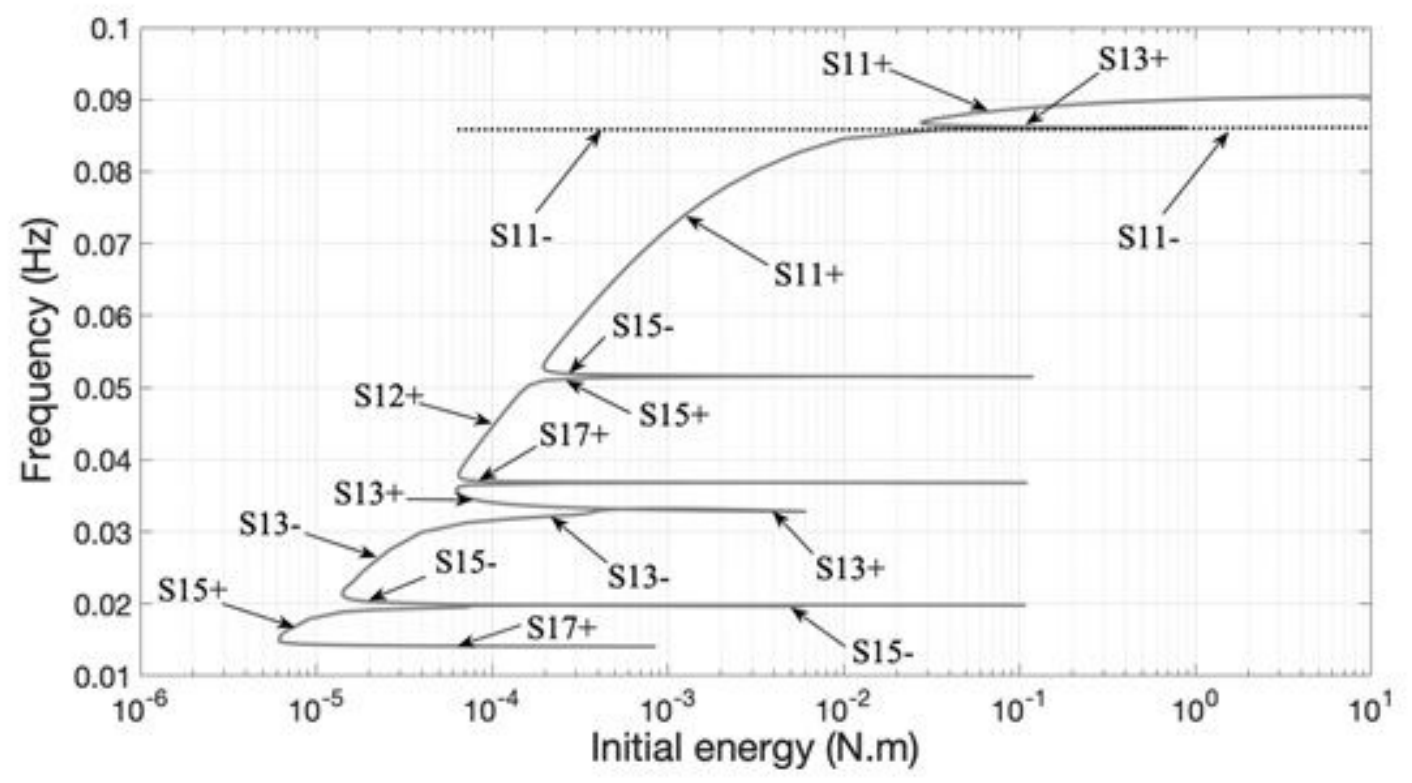

Figure 2

The lowest frequency backbone curve and its bifurcated subharmonic branches of symmetric in phase and anti-phase periodic motions on the NNMs. 

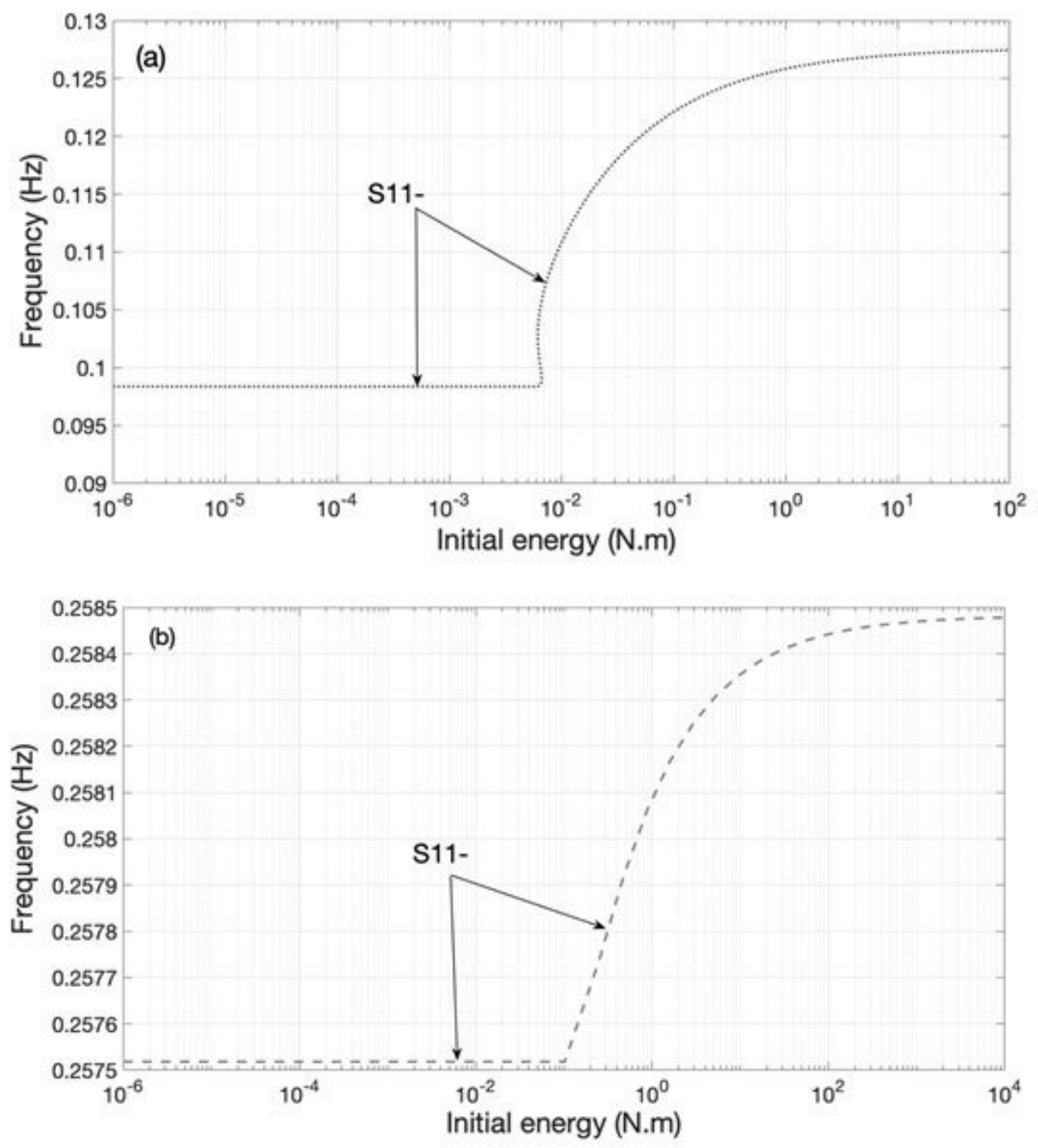

\section{Figure 3}

The antiphase intermediate frequency backbone curve in (a) and the higher frequency antiphase backbone curve in (b) of the periodic motions on the NNMs. 


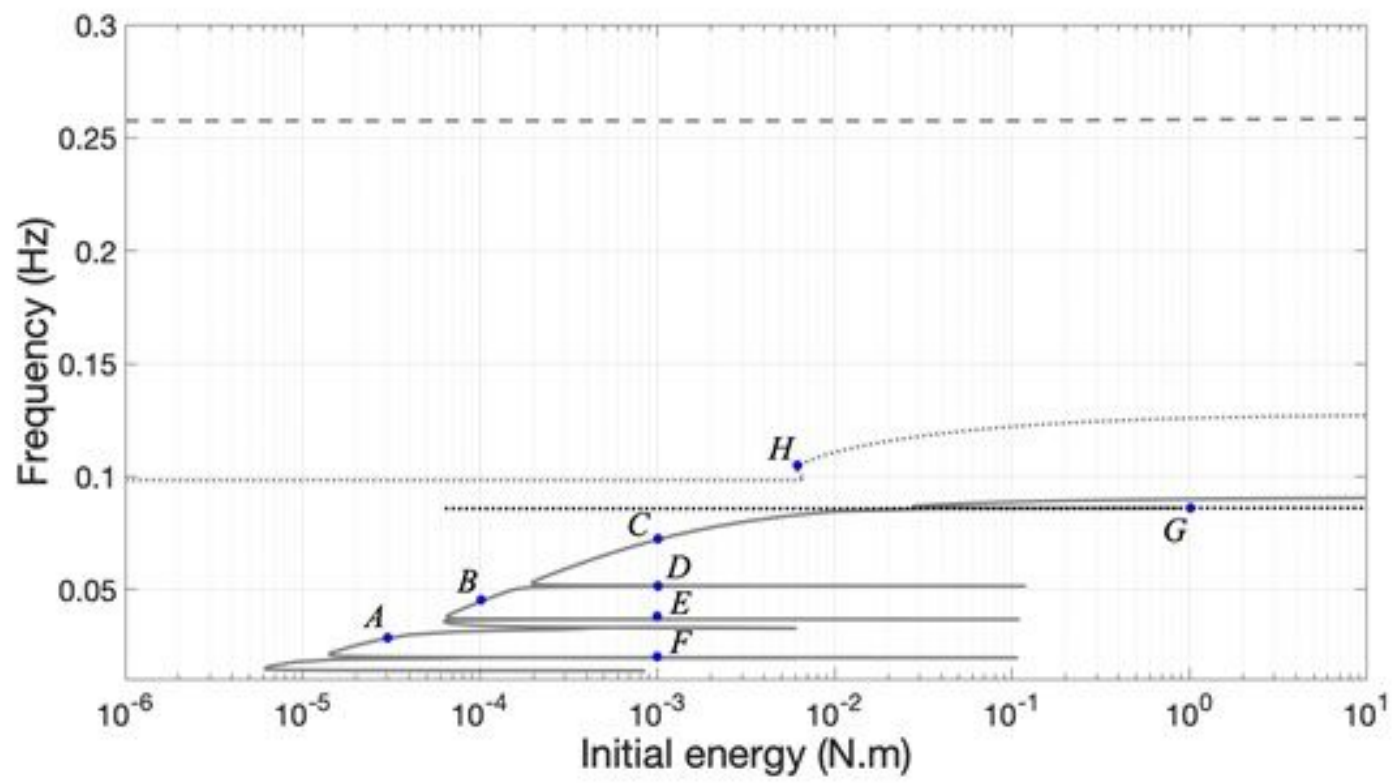

\section{Figure 4}

The three obtained backbone curves of the FEP of the structure-PNES system and their associated internal resonance subharmonic branches. 

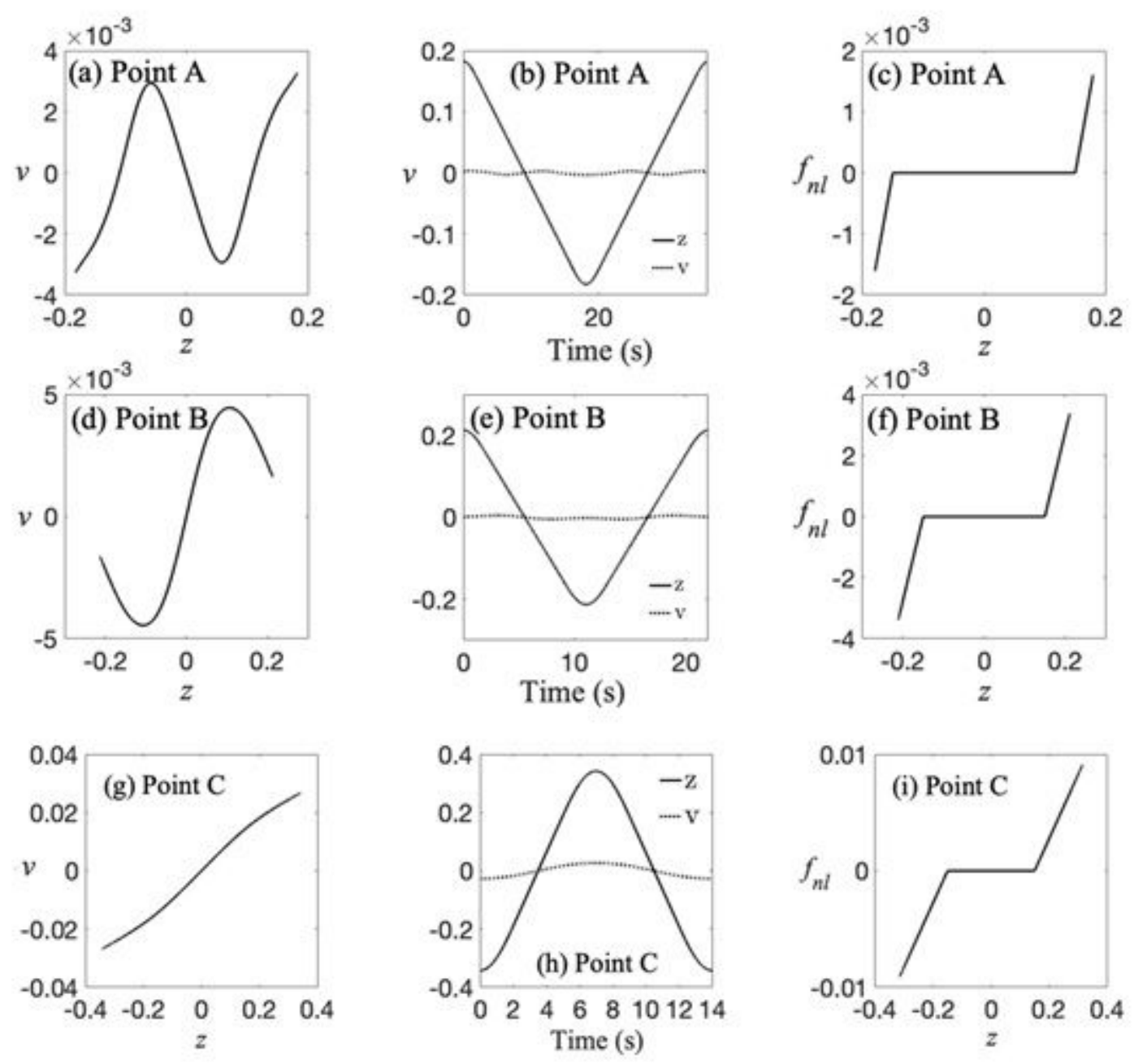

\section{Figure 5}

The NNMs in (a), (d) and (g), their corresponding response of the structure-PNES system in (b), (e) and (h), and the corresponding nonlinear NES coupling forces in (c), (f) and (i) for the data points A, B and C in the FEP plot in Fig. 4. 

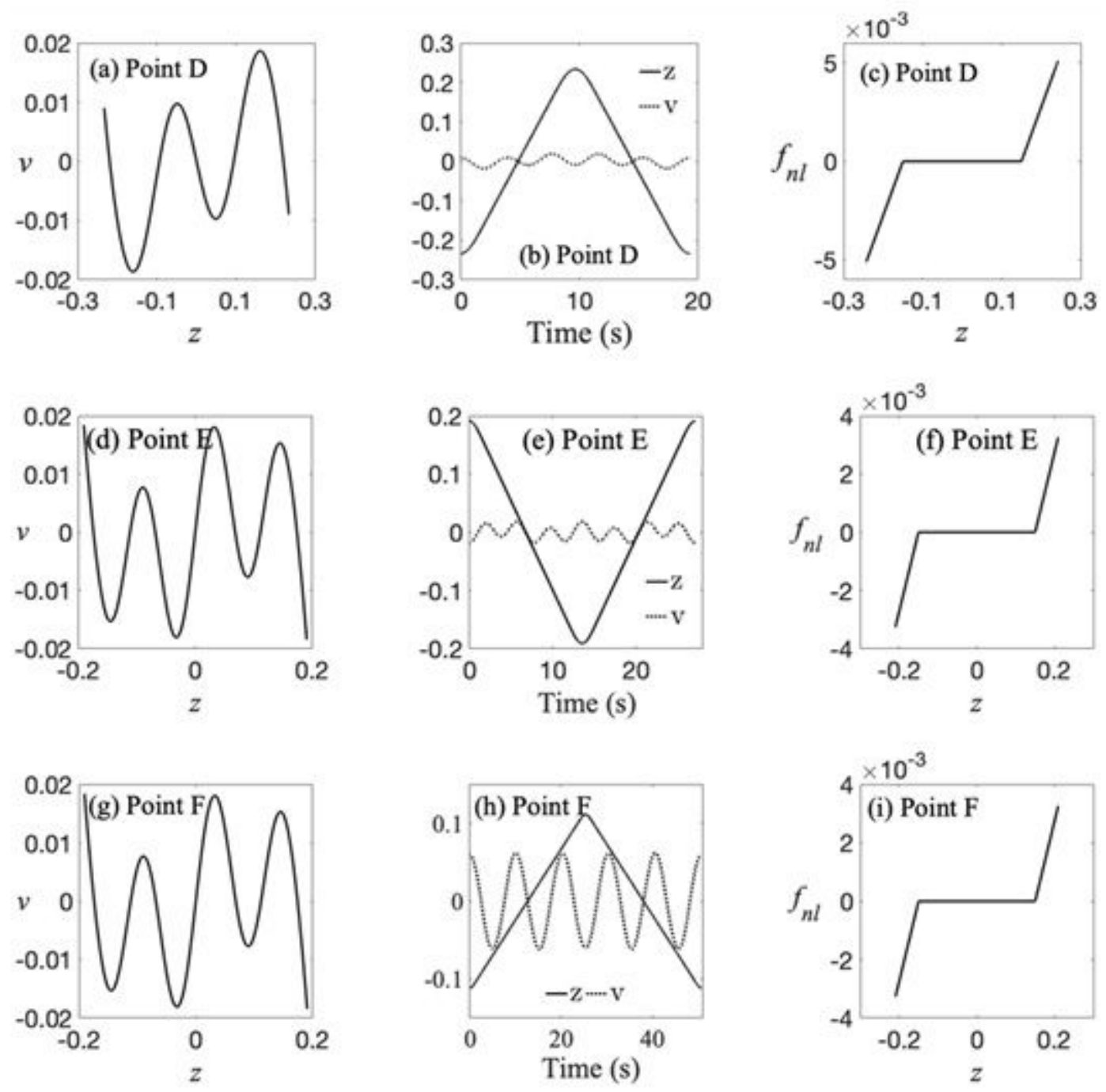

\section{Figure 6}

The NNMs in (a), (d) and (g), their corresponding response of the structure-PNES system in (b), (e) and (h), and the corresponding nonlinear NES coupling forces in (c), (f) and (i) for the data points D, E and $F$ in the FEP plot in Fig. 4. 

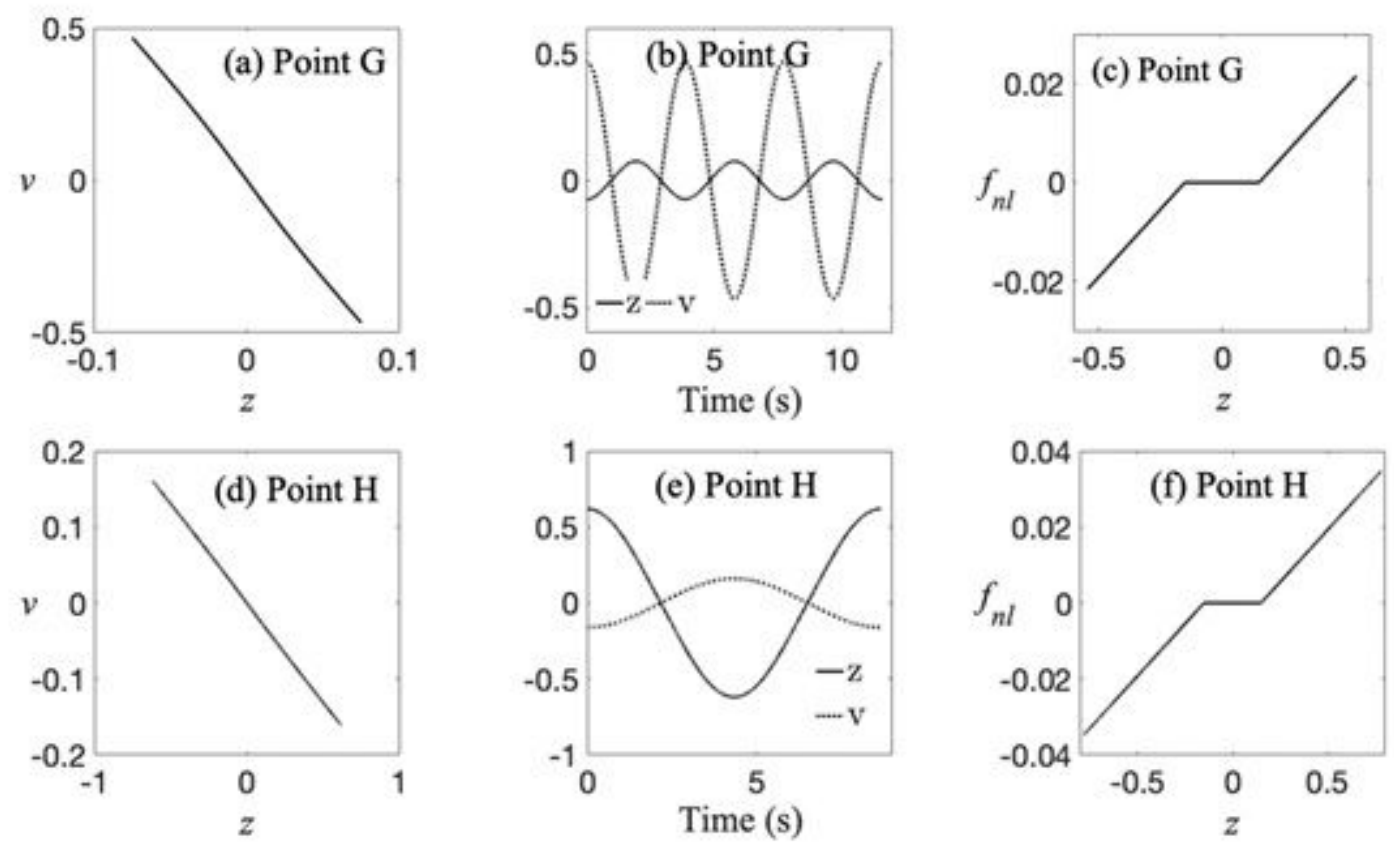

\section{Figure 7}

. The antiphase NNMs in (a) and (d), their corresponding response of the structure-PNES system in (b) and (e), and the corresponding nonlinear NES coupling forces in (c) and (f) for the data points $\mathrm{G}$ and $\mathrm{H}$ in the FEP plot in Fig. 2.
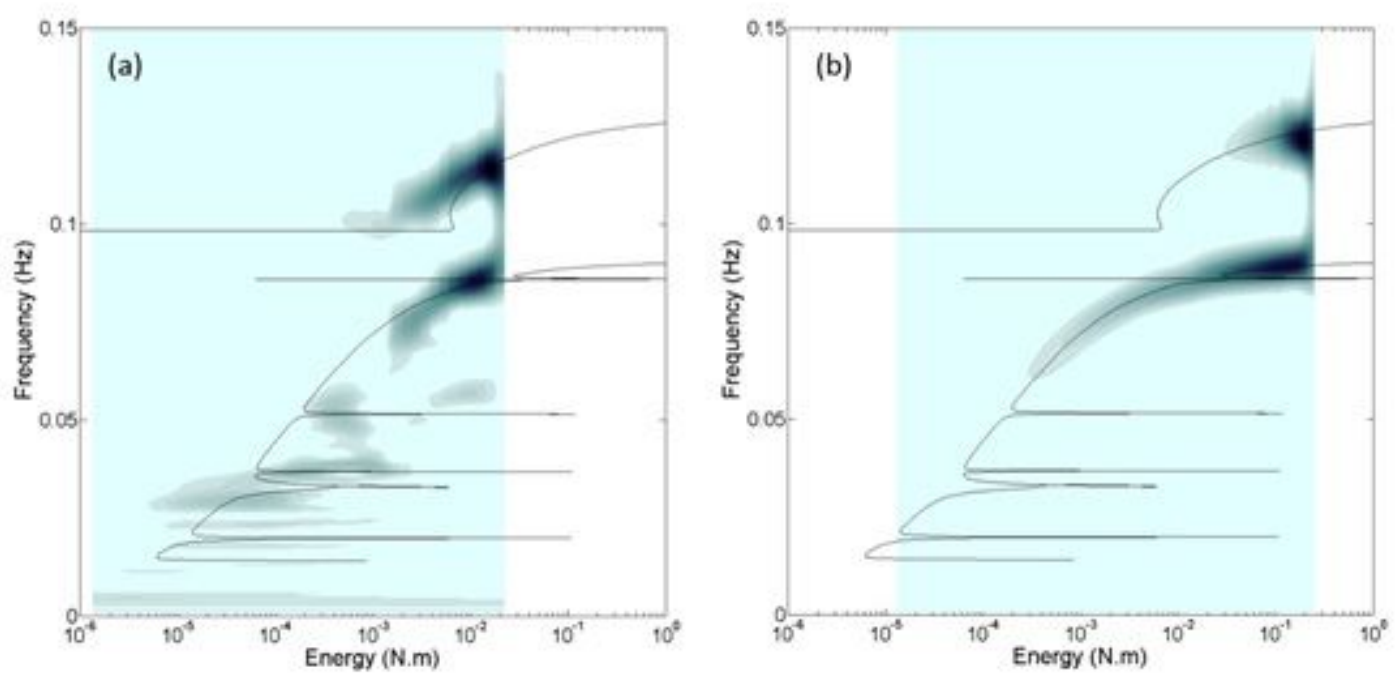

\section{Figure 8}

Imposed wavelet transform frequency spectrum on the FEP of the damped response of the structurePNES system in (a) at $u(0)$ and $v(0)=1.5 \mathrm{~m} / \mathrm{s}$ in $(\mathrm{b}) \mathrm{u}(0)$ at $v(0)=0.5 \mathrm{~m} / \mathrm{s}$. 


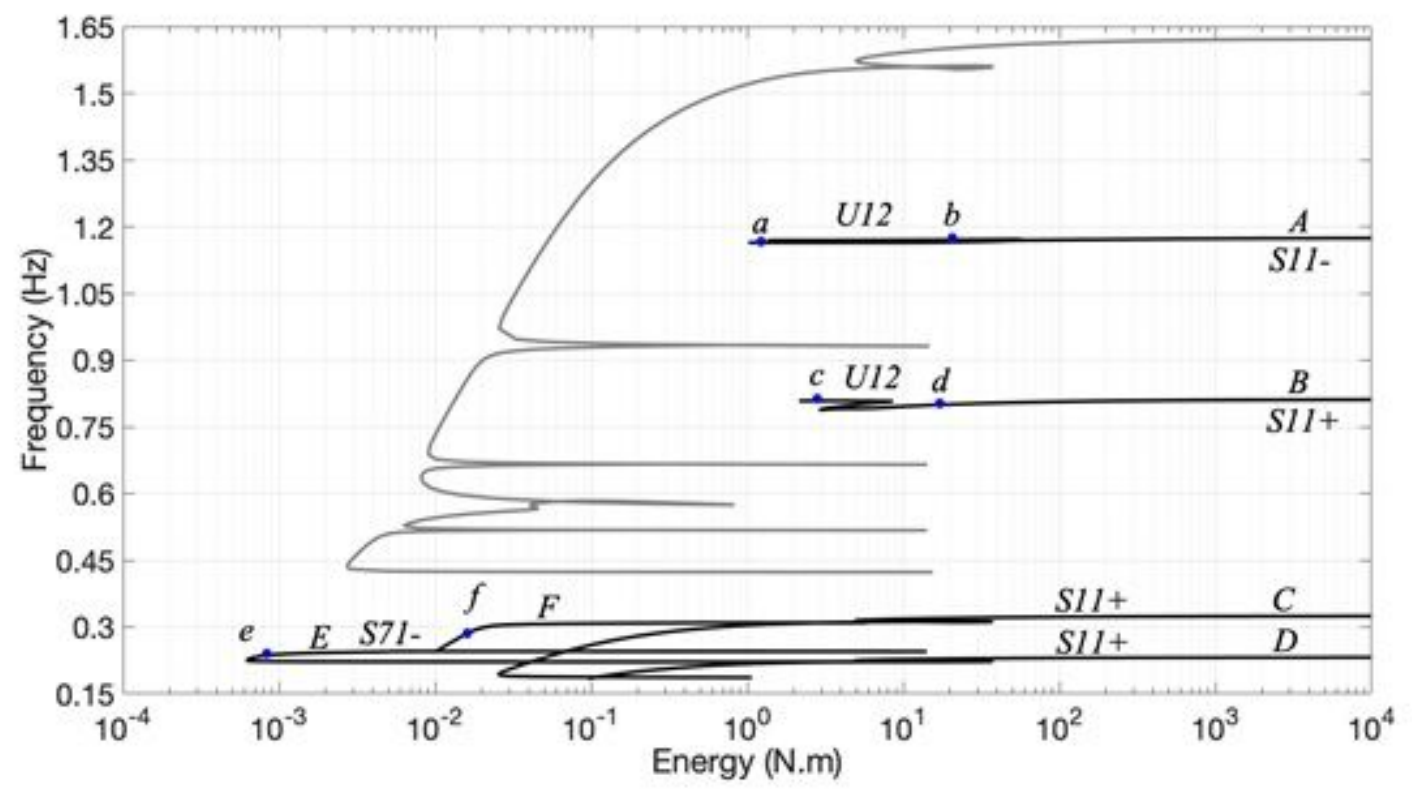

\section{Figure 9}

The lowest frequency backbone curve and its associated subharmonic branches and tongues of symmetrical and unsymmetrical NNMs periodic motions.
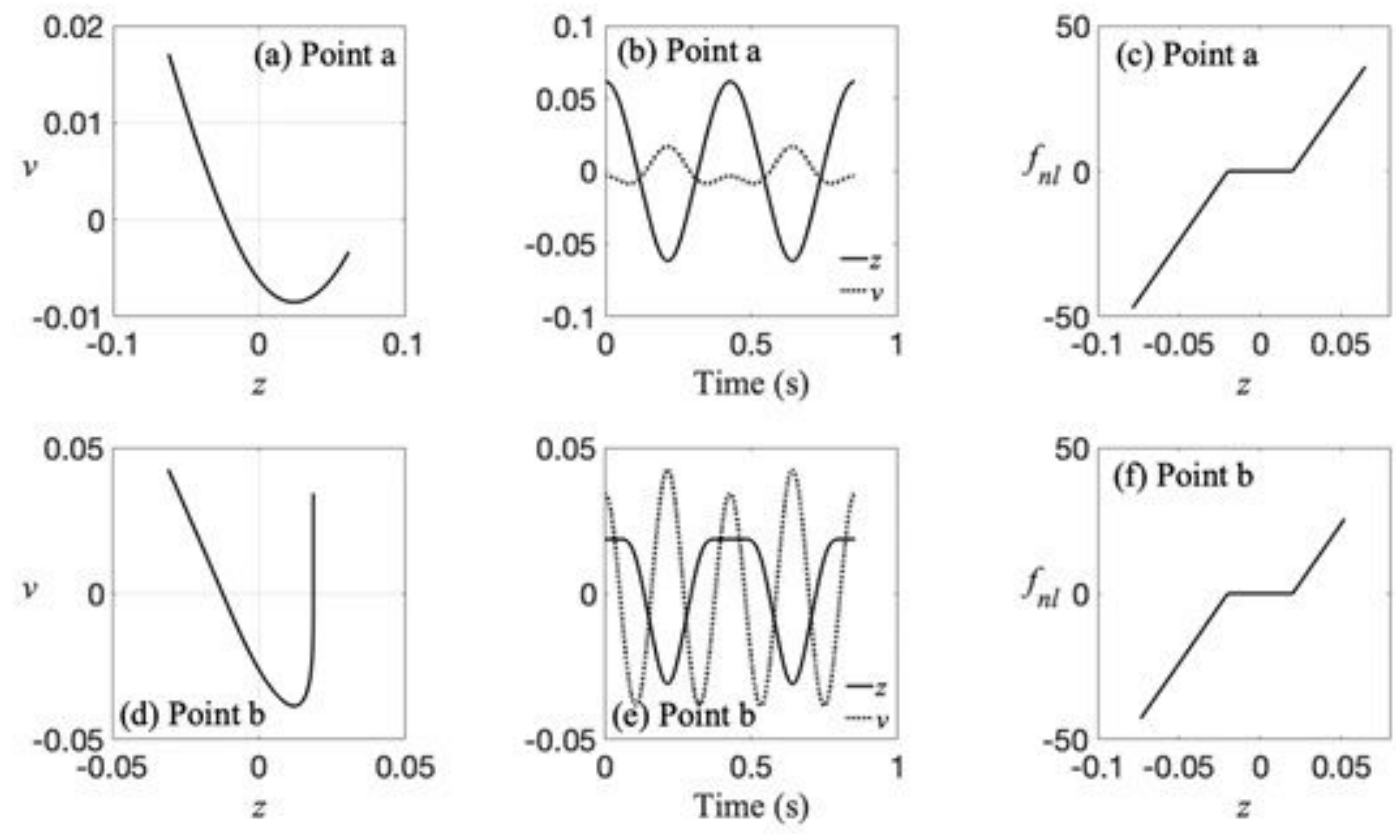

Figure 10

The antiphase NNMs in (a) and (d), their corresponding response of the structure-PNES system in (b) and (e), and the corresponding nonlinear NES coupling forces in (c) and (f) for the data points $a$ and $b$ in the FEP plot in Fig. 9. 

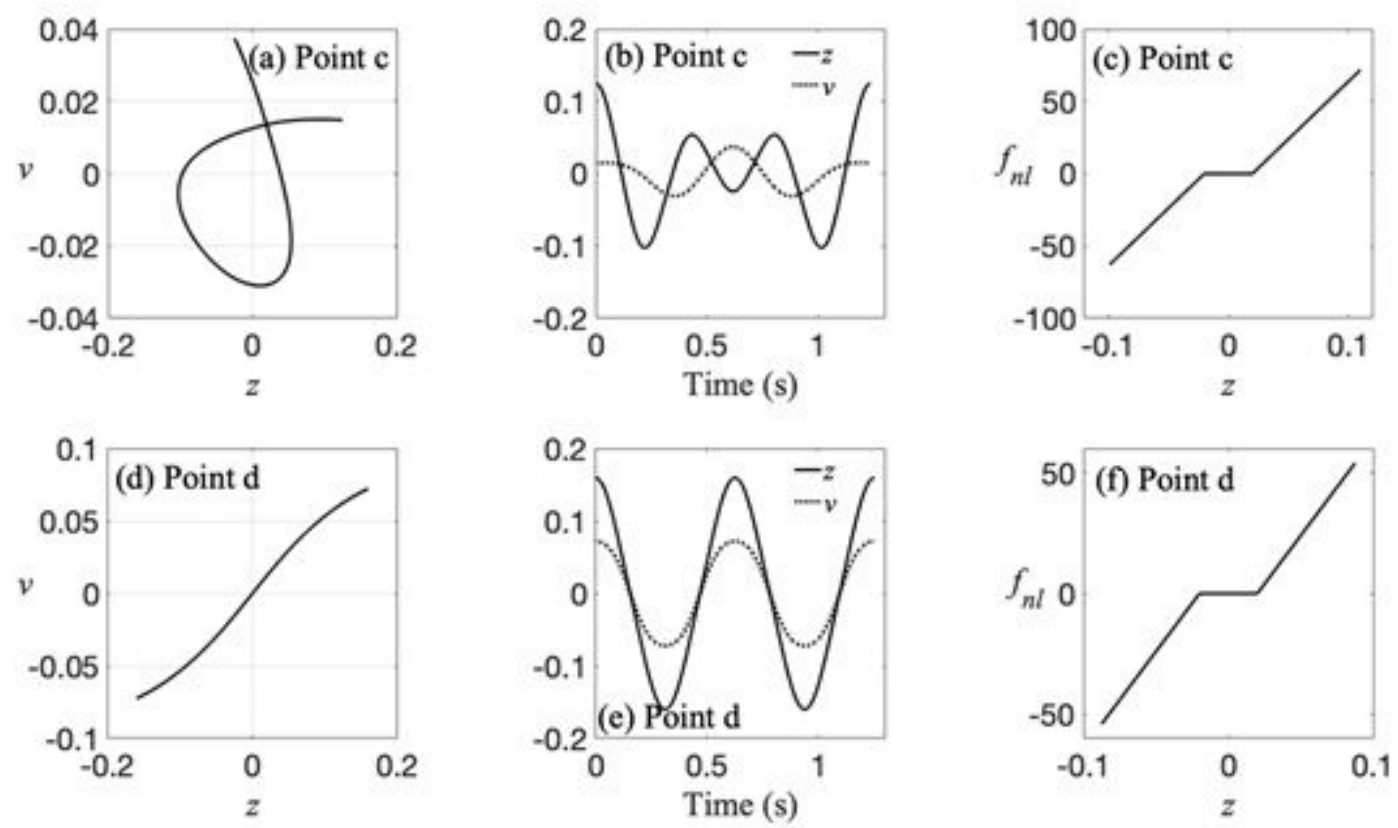

\section{Figure 11}

The antiphase NNMs in (a) and (d), their corresponding response of the structure-PNES system in (b) and (e), and the corresponding nonlinear NES coupling forces in (c) and (f) for the data points $c$ and $d$ in the FEP plot in Fig. 9.
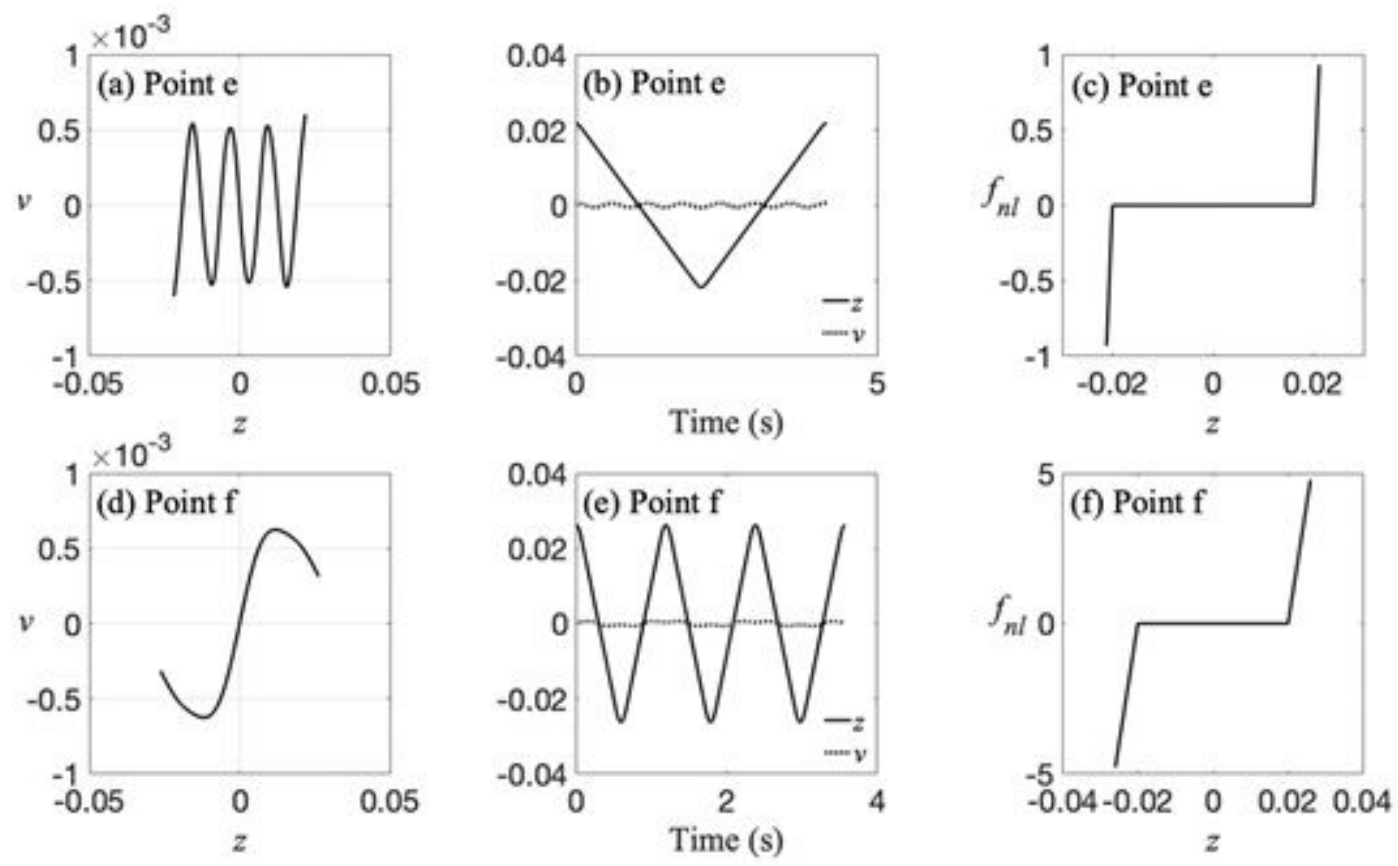

\section{Figure 12}

The antiphase NNMs in (a) and (d), their corresponding response of the structure-PNES system in (b) and (e), and the corresponding nonlinear NES coupling forces in (c) and (f) for the data points e and $f$ in the 
FEP plot in Fig. 9.

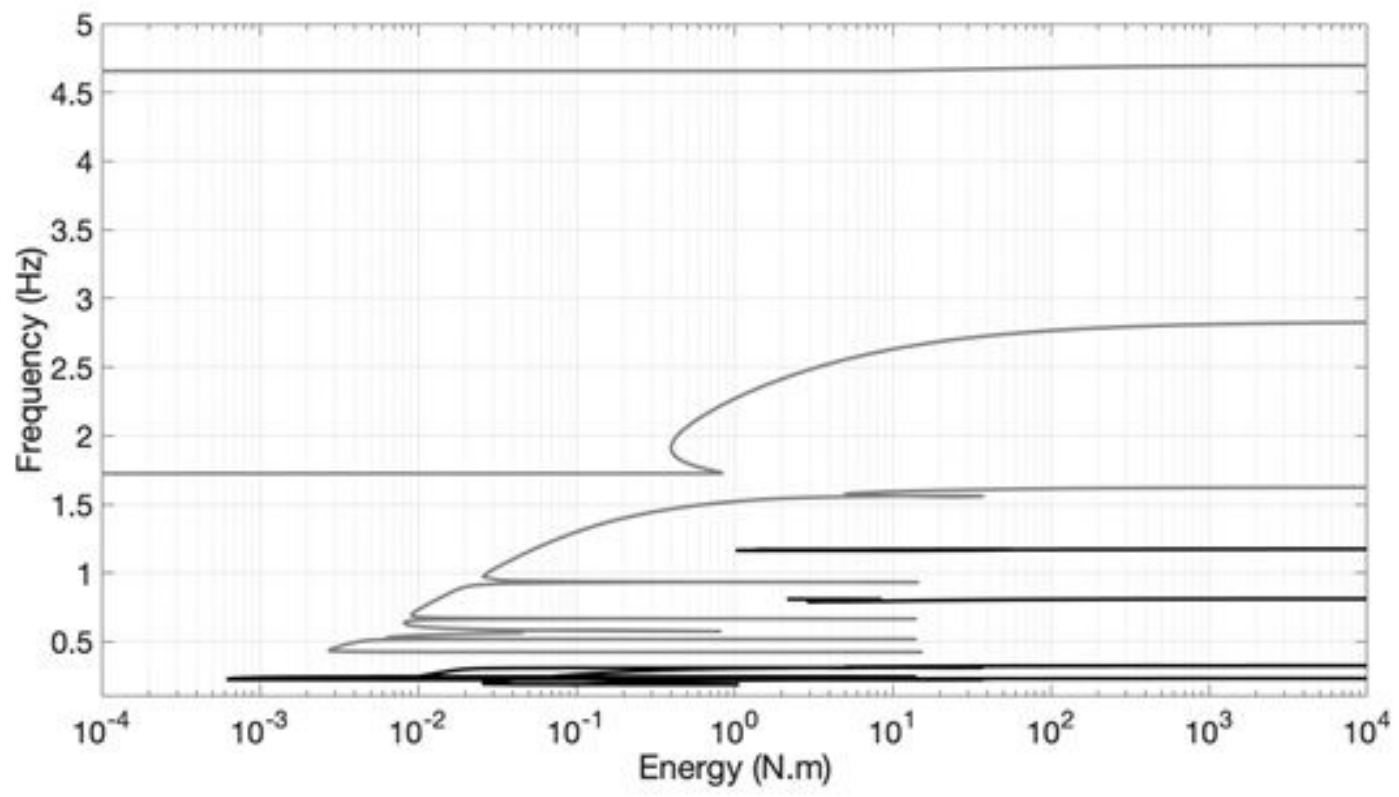

\section{Figure 13}

The three obtained backbone curves of the FEP of the structure-PNES system and the associated subharmonic branches of the NNMs periodic motion. 

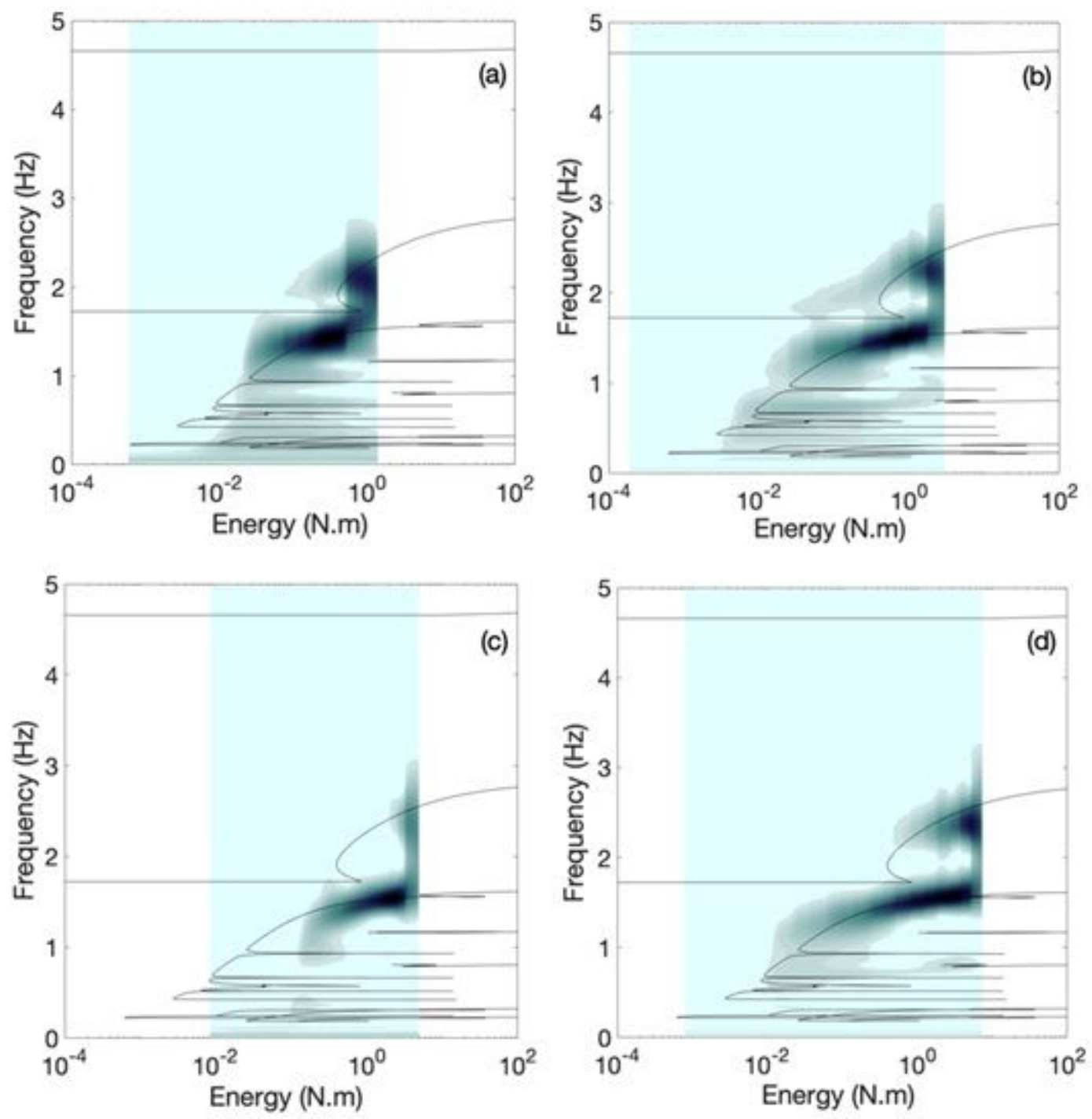

Figure 14

Imposed wavelet transform frequency spectrum on the FEP of the damped response of the structurePNES system. 